\title{
Construction of fastest curvature-constrained paths in direction-dependent media
}

\author{
Alvaro Maggiar*and Irina S. Dolinskaya ${ }^{\dagger}$ \\ Northwestern University, Evanston, IL 60201
}

\begin{abstract}
This paper presents an algorithm that constructs a fastest curvature-constrained path in a directiondependent environment for a given initial and target locations and heading angles. The problem studied here is a generalization of the classical Dubins car problem, where the vehicle speed and minimumturning radius are assumed to be constant. This assumption is relaxed and the settings where the two parameters are arbitrary functions of the agent's heading angle are considered, such as a maneuvering sailboat for example. This paper is concerned with the extension and implementation of the authors' earlier results that establish the fastest path between two positions in the plane for a Dubins-like vehicle in a (possibly) anisotropic medium to be of the form $\mathcal{C S C S C}$ - or any subset of this word - where $\mathcal{C}$ denotes a sharpest turn and $\mathcal{S}$ a straight-line segment. While the authors' preceding work has derived the structure of a fastest path, the actual implementation of the results presents a significant challenge and remained unsolved. The main contribution of this paper is an algorithm that implements those results and illustrates several specific instances in which the results developed here can be applied. This work is particularly relevant for vehicles whose interaction with their surrounding environment creates direction-dependent dynamics, such as aerial or surface vehicles in wind or strong currents.
\end{abstract}

\section{Introduction}

This paper studies the problem of finding fastest curvature-constrained paths in direction-dependent media. This is a generalization of the classical Dubins car problem Dubins, 1957], by relaxing the assumption of constant speed and minimum-turning radius of a vehicle and considering the settings where the two parameters are arbitrary functions of the agent's heading angle. This paper is concerned with the extension and implementation of the results derived in Dolinskaya and Maggiar 2012. Dolinskaya and Maggiar proved that the fastest path between two positions in the plane for a Dubins-like vehicle in a (possibly) anisotropic medium is of the form $\mathcal{C S C S C}$ - or any subset of this word - where $\mathcal{C}$ denotes a sharpest turn and $\mathcal{S}$ a straightline segment. It was also shown that in the particular case of a convex speed polar plot (i.e., a speed function whose enclosed area when plotted in polar coordinates is convex), there exists a fastest path of a structure similar to the classical Dubins path, i.e., $\mathcal{C S C}$ or $\mathcal{C C C}$. While Dolinskaya and Maggiar derived the structure of a fastest path, the actual implementation of the result is challenging for reasons which are expanded on below and remained unsolved. The aim of this paper is to construct an algorithm that implements those results and to illustrate several particular instances in which the results developed therein can be applied. One of the main contributions of this paper is Algorithm 1. which, given direction dependent speed and minimum-turning radius functions, computes the fastest path between an initial and a final configurations.

\subsection{Motivation and related literature}

The problem of finding fastest paths for vehicles whose motion is constrained by a minimum-turning radius is of great practical interest since many vehicles, in particular robots, fall into this category. One of the

*PhD candidate, Department of Industrial Engineering and Management Sciences.

${ }^{\dagger}$ Assistant Professor, Department of Industrial Engineering and Management Sciences. 
most common models is that of a car-like vehicle whose controls are the speed and the angular velocity. In a seminal paper, Dubins 1957 considered a vehicle with isotropic and constant speed and minimum-turning radius, and showed via geometrical arguments that the structure of a fastest path between an initial and a final configuration was of the form $\mathcal{C S C}$ or $\mathcal{C C C}$ - or any subset of those. Reeds and Shepp 1990 considered a broader model in which the vehicle is allowed to move both forward and backward and derived an analogous structure for time-optimal paths. The results obtained by Dubins [1957] and Reeds and Shepp 1990] were later re-derived using optimal control theory arguments concurrently by Sussmann and Tang [1991] and Boissonnat et al. [1994]. Bui et al. [1994] then provided a synthesis of optimal paths in the plane for the Dubins problem - i.e., a partition of the plane into regions of similar optimal structure - while Soueres and Boissonnat 1998 carried out this task for the Reeds and Shepp model. The optimal control theory method has enabled numerous extensions of the Dubins model, notably the case of a Dubins-like airplane moving in a wind-field. McGee et al. 2005] considered the case of a constant and uniform wind and proved that the solution of the problem still has a Dubins-path structure by recasting the problem as a moving target problem. The same idea was used by McNeely et al. 2007] to allow for a still space-uniform but time dependent wind-field. Still in the field of aerial vehicles, Hima and Bestaoui 2003] considered autonomous underactuated airships.

Motivated by current research regarding the optimal short-range routing of a vessel in a stationary random seaway Dolinskaya et al., 2009, Dolinskaya and Maggiar 2012 considered a more general case wherein both the speed and minimum-turning radius of the vehicle are anisotropic, and in which the speed polar plot is potentially non-convex. An example of such a motivating speed polar plot is displayed in Figure 1. This problem encompasses many of the previous extensions to the Dubins problem, such as the constant wind case, but also enables the study of other instances where the physical interaction of the vehicle with its surroundings yields a heading dependency of its speed and/or minimum-turning radius. Sailing ships, whose speed polar plot are non-convex, are one such example (see for instance Philpott and Mason [2001]). Dolinskaya and Maggiar 2012 showed that, in general, an optimal path is of the form $\mathcal{C S C S C}$, or any subset of this word, and that in the particular case of a convex speed polar plot, an optimal path had a Dubins-like $(\mathcal{C S C}$ or $\mathcal{C C C})$ structure. Concurrently and motivated by a very different problem dealing with the design of underground mine access in bad ground where the cost of digging depends on the characteristics of the rock and exhibits anisotropic properties, Chang et al. 2012 and Chang et al. 2011 derived similar results, although they restricted their analysis to a constant minimum-turning radius.

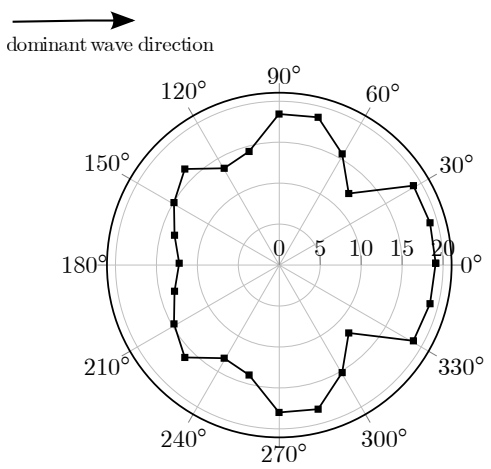

Figure 1: An example of speed polar plot for the S175 containership in Sea State 7 irregular wavefield (adapted from Dolinskaya et al. 2009)

The implementation of the Dubins and Reeds-Sharp results is usually carried out by trying all the candidate paths of the specified structure and selecting the fastest one. However, Wang et al. 2009 employed geometrical arguments based on results obtained from Pontryagin's maximum principle to derive a complete algorithm with a low computational cost. Techy and Woolsey 2009 developed an algorithm for the constant wind case that can be seen as a particular case of the algorithm for the convex case derived in Dolinskaya and Maggiar 2012. In the study of the problem with anisotropic speed function but constant minimum-turning radius, Chang 2011] implemented an algorithm for piecewise constant speed functions. This paper, on the other hand, does not make such restricting assumptions and develops an algorithm for general differentiable 
speed and minimum-turning radius functions. In addition, results of practical interest in the case of a piecewise linear speed polar plot are presented.

\subsection{Problem Statement}

The object of the problem is a point-mass vehicle whose motion is constrained by two functions having a domain $S^{1}=[0,2 \pi]$ and taking values in $\mathbb{R}^{+}$, the set of non-negative real numbers. These two functions are:

speed function : $V: S^{1} \rightarrow \mathbb{R}^{+}$that characterizes the maximum speed attainable by the vehicle along a direction heading $\theta$,

minimum-turning radius function : $R: S^{1} \rightarrow \mathbb{R}^{+}$that specifies the dependence of the vehicle's minimumturning radius depending on its heading.

Note that in many applications, the two functions are not independent for the minimum-turning radius is often itself a function of the speed.

The vehicle has a planar motion and its state is perfectly characterized by a configuration $(x, y, \theta) \in$ $\mathcal{M}=\mathbb{R}^{2} \times S^{1}$, where $(x, y)$ is the position of the vehicle in the plane and $\theta$ its heading.

To characterize the dynamics of the vehicle, let $t$ index the time and define the functions $x(t), y(t)$ and $\theta(t)$ in an obvious manner to represent the state of the vehicle at time $t$. The motion of the vehicle can be altered through a steering control $u(t)$ that represents the rate of change of the vehicle's heading at time $t$. The differential system governing the dynamics of the vehicle then reads:

$$
\begin{aligned}
& \dot{x}=V(\theta) \cos \theta, \\
& \dot{y}=V(\theta) \sin \theta, \\
& \dot{\theta}=\frac{V(\theta)}{R(\theta)} u .
\end{aligned}
$$

For a more detailed discussion, see Dolinskaya and Maggiar 2012].

This paper is concerned with the problem of finding a fastest path between an initial configuration $\left(x_{s}, y_{s}, \theta_{s}\right)$ and a final configuration $\left(x_{t}, y_{t}, \theta_{t}\right)$. It will be assumed throughout the paper and without loss of generality that the vehicle is initially located at the origin, so that $\left(x_{s}, y_{s}\right)=(0,0)$.

\subsection{Structure of the paper}

The paper is structured as follows: in Section 2 additional necessary properties satisfied by optimal paths are derived by writing the fastest-path problem as an optimization problem. These properties enable in Section 3 the derivation of an algorithm that solves the fastest-path finding problem for differentiable speed functions. In Section 4 numerical results obtained by applying the algorithms derived in the paper are presented. Finally, section 5 concludes the paper and presents some axis of further development and future research.

\section{Additional properties of an optimal path}

As proved in Dolinskaya and Maggiar 2012, there exists an optimal path for the problem of the form $\mathcal{C S C S C}$, or any subset of this word. The result, however, does not provide any information as to what the structure actually is for a given initial and final configuration, let alone what the path itself is. The same kind of difficulty raised by Wang et al. 2009 is faced:

"No method can provide the sufficient conditions of determining optimal trajectories for the kinematic models of wheeled robots, but only the necessary conditions can be achieved. Unfortunately, these necessary conditions still have not provided enough information for the determination of the optimal trajectories so that more tools are needed to be exploited for the solutions. Therefore, in general, the solution of such problems involves three steps: 1) searching a sufficient family of optimal trajectories; 2) achieving an optimal trajectory synthesis; and 3) obtaining an optimal algorithm." 
This paper thus proceeds in a similar fashion, by first deriving additional properties of the family of optimal trajectories. In order to do so, and similarly to Chang [2011], it is observed that the optimal paths of the problem can be divided into three categories:

Dubins-like paths: these are of the form $\mathcal{C C C}$ or $\mathcal{C S C}$ (and their degeneracies).

$\mathcal{S C C}$ paths: Paths containing a non-degenerate sub-path of the form $\mathcal{S C C}$ or $\mathcal{C C S}$.

$\mathcal{S C S}$ paths: Paths containing a non-degenerate sub-path of the form $\mathcal{S C S}$.

The first objective is to reduce the size of the family of candidate optimal paths. The first type of optimal paths can be dealt with using the algorithm presented in Dolinskaya and Maggiar 2012 for the convex speed polar plot case, which are elaborated on in Section 3.1. The next sections thus focus on the $\mathcal{S C C}$ and $\mathcal{S C S}$ paths so as to establish further properties they satisfy. Writing the optimization problem solved by an optimal path, it is possible to derive local information giving necessary conditions for optimality, which combined with additional geometrical insight allow to state more precise propositions about the characteristics of $\mathcal{S C C}$ and $\mathcal{S C S}$ paths.

\section{$2.1 \quad$ Optimization model}

In this section, the results derived in Dolinskaya and Maggiar 2012 are used to write the fastest path problem as an optimization problem.

\subsubsection{Notations and definitions}

Before delving into the actual formulation of the optimization problem, the notations and functions used are clarified and defined, many of which are adapted from Dolinskaya and Maggiar 2012. The problem requires the derivation of displacements and travel times along sharpest-turn curves for which corresponding functions are defined. It is recalled that in Dolinskaya and Maggiar 2012, the following terminology is introduced to describe the elements of a path:

$\mathcal{C}_{R}\left(\theta_{1}, \theta_{2}\right)\left(\mathcal{C}_{L}\left(\theta_{1}, \theta_{2}\right)\right)$ : a continuous segment (i.e., arc) of the right-hand (left-hand) sharpest turn curve that starts at the heading angle $\theta_{1}$, ends at the angle $\theta_{2}$, and spans an interval of headings smaller than $2 \pi$ (i.e., the vehicle does not make a complete $2 \pi$-turn). The curve is defined for $\theta_{1}, \theta_{2} \in S^{1}$. Consequently, if $\theta_{2}>\theta_{1}$ for $\mathcal{C}_{R}\left(\theta_{1}, \theta_{2}\right)$ it is assumed that the curve ends at the angle $\theta_{2}-2 \pi$, and if $\theta_{1}>\theta_{2}$ for $\mathcal{C}_{L}\left(\theta_{1}, \theta_{2}\right)$ that the curve ends at the angle $\theta_{2}+2 \pi$.

$\Theta_{R}\left(\theta_{1}, \theta_{2}\right)\left(\Theta_{L}\left(\theta_{1}, \theta_{2}\right)\right)$ : the set of all headings taken by an agent traversing a right-hand (left-hand) sharpest turn curve $\mathcal{C}_{R}\left(\theta_{1}, \theta_{2}\right)\left(\mathcal{C}_{L}\left(\theta_{1}, \theta_{2}\right)\right)$. Since the heading angle cannot be changed instantaneously, it is natural for the set of spanned angles to be a continuous interval of heading angles. For consistency, assume that $\Theta_{R}\left(\theta_{1}, \theta_{2}\right) \bigcup \Theta_{L}\left(\theta_{1}, \theta_{2}\right) \subseteq S^{1}$. Consequently,

$$
\Theta_{R}\left(\theta_{1}, \theta_{2}\right):=\left\{\begin{array}{ll}
{\left[\theta_{2}, \theta_{1}\right],} & \text { if } \theta_{1}>\theta_{2} \\
{\left[0, \theta_{1}\right] \cup\left[\theta_{2}, 2 \pi\right),} & \text { if } \theta_{2}>\theta_{1} \\
\theta_{1} & \text { if } \theta_{1}=\theta_{2}
\end{array} \text { and } \Theta_{L}\left(\theta_{1}, \theta_{2}\right):=\left\{\begin{array}{ll}
{\left[\theta_{1}, \theta_{2}\right],} & \text { if } \theta_{1}<\theta_{2} \\
{\left[0, \theta_{2}\right] \cup\left[\theta_{1}, 2 \pi\right),} & \text { if } \theta_{2}<\theta_{1} \\
\theta_{1} & \text { if } \theta_{1}=\theta_{2}
\end{array} .\right.\right.
$$

$\left\|\Theta_{k}\left(\theta_{1}, \theta_{2}\right)\right\|$ : size of the set $\Theta_{k}\left(\theta_{1}, \theta_{2}\right)(k \in\{R, L\})$, which is equal to the sum of the lengths of the angle intervals belonging to the set.

$D($.$) : a displacement vector (from the start point to the end point) for a path given as an input. For$ example, $D\left(\mathcal{C}_{R}\left(\theta_{1}, \theta_{2}\right)\right)$ denotes the displacement vector corresponding to a curve $\mathcal{C}_{R}\left(\theta_{1}, \theta_{2}\right)$.

Further define the components of $D$ as $D^{x}$ and $D^{y}$, corresponding to the $x$-axis and $y$-axis displacements so that $D()=.\left(D^{x}(.), D^{y}(.)\right)^{T}$.

Of particular interest is the displacement along a sharpest turn from a heading $\theta_{a}$ to a heading $\theta_{b}$ :

$$
D\left(\mathcal{C}_{k}\left(\theta_{a}, \theta_{b}\right)\right)=\left(\begin{array}{l}
\int_{\Theta_{k}\left(\theta_{a}, \theta_{b}\right)} R(\theta) \cos \theta d \theta \\
\int_{\Theta_{k}\left(\theta_{a}, \theta_{b}\right)} R(\theta) \sin \theta d \theta
\end{array}\right),
$$


where $k \in\{R, L\}$.

$\beta($.$) : a heading angle of a vector specified as an input. For examples \beta\left(D_{s t}\right)$ denotes the angle of the displacement vector $D_{s t}$ from $\left(x_{s}, y_{s}\right)$ to $\left(x_{t}, y_{t}\right)$.

$\tau($.$) : a travel time function that returns the total travel time along a path specified as an input. For$ example, $\tau\left(\mathcal{C}_{R}\left(\theta_{1}, \theta_{2}\right)\right)$ denotes the travel time along a curve $\mathcal{C}_{R}\left(\theta_{1}, \theta_{2}\right)$.

The value of the travel time function can be computed as follows:

sharpest turn curve $\mathcal{C}_{k}\left(\theta_{a}, \theta_{b}\right): \quad($ where $k \in\{R, L\})$

$$
\tau\left(\mathcal{C}_{k}\left(\theta_{a}, \theta_{b}\right)\right)=\left|\int_{\Theta_{k}\left(\theta_{a}, \theta_{b}\right)} \frac{R(\theta)}{V(\theta)} d \theta\right|=u \int_{\Theta_{k}\left(\theta_{a}, \theta_{b}\right)} \frac{R(\theta)}{V(\theta)} d \theta,
$$

where $u=-1$ if $k=R$ and $u=1$ if $k=L$.

straight line segment from $a=\left(x_{a}, y_{a}\right)$ to $b=\left(x_{b}, y_{b}\right)$ (denoted by $\left.S_{a b}\right): \quad \tau\left(S_{a b}\right)=\frac{\|\overrightarrow{a b}\|}{V\left(\beta\left(S_{a b}\right)\right)}$.

Let $T\left(\theta_{1}, \theta_{2}, u\right)$ be a function that outputs the travel time along a left or right turn from a heading $\theta_{1}$ to a heading $\theta_{2}$ depending on whether $u=+1$ or $u=-1$, respectively. $T$ can be written more explicitly as $T\left(\theta_{1}, \theta_{2}, u\right):=\left(\frac{u+1}{2}\right) \tau\left(\mathcal{C}_{L}\left(\theta_{1}, \theta_{2}\right)\right)+\left(\frac{1-u}{2}\right) \tau\left(\mathcal{C}_{R}\left(\theta_{1}, \theta_{2}\right)\right)$. This expression will be useful in writing out the optimization problem because it makes the function $T$ differentiable with respect to $u$. Furthermore, observe that $\frac{\partial \tau\left(\mathcal{C}_{L}\left(\theta_{1}, \theta_{2}\right)\right)}{\partial \theta_{1}}=-\frac{R\left(\theta_{1}\right)}{V\left(\theta_{1}\right)}$ and $\frac{\partial \tau\left(\mathcal{C}_{R}\left(\theta_{1}, \theta_{2}\right)\right)}{\partial \theta_{1}}=+\frac{R\left(\theta_{1}\right)}{V\left(\theta_{1}\right)}$, which yields $\frac{\partial T}{\partial \theta_{1}}\left(\theta_{1}, \theta_{2}, u\right)=-u \frac{R\left(\theta_{1}\right)}{V\left(\theta_{1}\right)}$. Likewise, one can find $\frac{\partial T}{\partial \theta_{2}}\left(\theta_{1}, \theta_{2}, u\right)=u \frac{R\left(\theta_{2}\right)}{V\left(\theta_{2}\right)}$, so that:

$$
\nabla_{\boldsymbol{\theta}} T\left(\theta_{1}, \theta_{2}, u\right)=u\left(-\frac{R\left(\theta_{1}\right)}{V\left(\theta_{1}\right)} \frac{R\left(\theta_{2}\right)}{V\left(\theta_{2}\right)}\right)^{T}
$$

Similarly, consider the functions $P_{x}\left(\theta_{1}, \theta_{2}, u\right):=\left(\frac{u+1}{2}\right) D^{x}\left(\mathcal{C}_{L}\left(\theta_{1}, \theta_{2}\right)\right)+\left(\frac{1-u}{2}\right) D^{x}\left(\mathcal{C}_{R}\left(\theta_{1}, \theta_{2}\right)\right)$ and $P_{y}\left(\theta_{1}, \theta_{2}, u\right):=$ $\left(\frac{u+1}{2}\right) D^{y}\left(\mathcal{C}_{L}\left(\theta_{1}, \theta_{2}\right)\right)+\left(\frac{1-u}{2}\right) D^{y}\left(\mathcal{C}_{R}\left(\theta_{1}, \theta_{2}\right)\right)$ which correspond to the $x$-axis and $y$-axis displacements, respectively, of a left $(u=1)$ or right $(u=-1)$ turn:

$$
\nabla_{\boldsymbol{\theta}} P_{x}\left(\theta_{1}, \theta_{2}, u\right)=u\left(\begin{array}{c}
-R\left(\theta_{1}\right) \cos \theta_{1} \\
R\left(\theta_{2}\right) \cos \theta_{2}
\end{array}\right), \quad \quad \nabla_{\boldsymbol{\theta}} P_{y}\left(\theta_{1}, \theta_{2}, u\right)=u\left(\begin{array}{c}
-R\left(\theta_{1}\right) \sin \theta_{1} \\
R\left(\theta_{2}\right) \sin \theta_{2}
\end{array}\right) .
$$

In Sections 2 and 3 , properties directly related to the local behavior of the speed polar plot at a given angle $\theta$ are derived and used. The interest will be whether the polar plot of $V$, which encloses a set defined as $\mathcal{V}$, is locally convex or concave, and whether the point $(\theta, V(\theta))$ (in polar coordinates) also belongs to the boundary of the convex hull, $\operatorname{conv}(\mathcal{V})$, of $\mathcal{V}$. The convexity or concavity of the speed polar plot at $\theta \in S^{1}$ is given by the sign of the signed curvature $\kappa(\theta)$ : if $\kappa(\theta) \geq 0$ the speed polar is convex at $\theta$ and concave otherwise. Let $\partial \mathcal{V}$ and $\partial \operatorname{conv}(\mathcal{V})$ be the boundaries of the speed polar plot and its convex hull, respectively (see Figure 2), an angle $\theta \in S^{1}$ is said to be a bordering angle if $(\theta, V(\theta)) \in \partial \operatorname{conv}(\mathcal{V}) \cap \partial \mathcal{V}$, a non-bordering convex angle if $\theta \in\{\theta:(\theta, V(\theta)) \notin(\partial \operatorname{conv}(\mathcal{V}) \cap \partial \mathcal{V}), \kappa(\theta) \geq 0\}$, and a non-bordering concave angle if $\theta \in\{\theta:(\theta, V(\theta)) \notin(\partial \operatorname{conv}(\mathcal{V}) \cap \partial \mathcal{V}), \kappa(\theta)<0\}$.

Finally, when a vehicle is moving in a non-uniform medium, as is the case in this paper, one should distinguish between the medium-relative orientation and the inertial orientation that results from the additional forces applied on the vehicle. The term heading will be used to refer to the medium-relative orientation and the term course to the inertial orientation.

\subsubsection{Problem Formulation}

Knowing the structure of an optimal path enables one to write down the problem of finding a fastest path as a minimization problem. It has been recalled that any optimal path is of the form $\mathcal{C S C S C}$ or any degenerate form of this word, meaning that it is allowed for some of the segments to be null. An optimal path is thus characterized by seven variables: $\left(\theta_{1}, \theta_{2}, l_{1}, l_{2}, u_{1}, u_{2}, u_{3}\right)$, where $\theta_{1}, \theta_{2} \in S^{1}, l_{1}, l_{2} \geq 0$ and $u_{1}, u_{2}, u_{3} \in\{-1,1\}$. These variables describe where the headings at which the turn switches or straight 


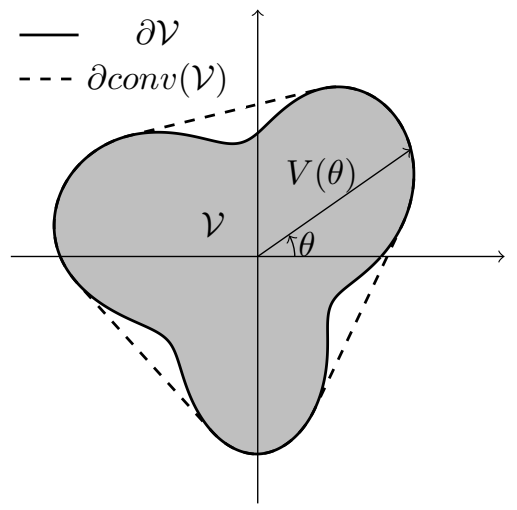

Figure 2: Illustration of the notations.

line occur, the lengths of the straight lines and the sense of the turns (left or right). More specifically, $\theta_{1}$ is the heading at which the first turn ends and at which there might be a straight line segment of length $l_{1}$; similarly $\theta_{2}$ is the heading at which the second turn ends and at which there might be a straight line segment of length $l_{2}$; and $u_{1}, u_{2}, u_{3}$ describe the orientation of the first, second and third turns, respectively, with -1 corresponding to a right turn and +1 to a left turn. Using this characterization of an optimal path, the travel time $f\left(\theta_{1}, \theta_{2}, l_{1}, l_{2}, u_{1}, u_{2}, u_{3}\right)$ of any candidate optimal path may be written as the sum of the travel times along each of the segments. For shortness of notation, the following will often be used: $\boldsymbol{\theta}:=\left(\theta_{1}, \theta_{2}\right)$, $\boldsymbol{l}:=\left(l_{1}, l_{2}\right), \boldsymbol{u}:=\left(u_{1}, u_{2}, u_{3}\right)$ and $\boldsymbol{z}:=(\boldsymbol{\theta}, \boldsymbol{l}, \boldsymbol{u})$.

The objective function to be minimized and the constraints by which the variables must abide can now be written. The objective function is the travel time along a candidate optimal path from $\left(0,0, \theta_{s}\right)$ to $\left(x_{t}, y_{t}, \theta_{t}\right)$ and, as pointed out, consists of the sum of the five possible segments it is made of: three curves and two straight lines with lengths $l_{1}$ and $l_{2}$. Thus, the objective function is:

$$
f(\boldsymbol{\theta}, \boldsymbol{l}, \boldsymbol{u})=T\left(\theta_{s}, \theta_{1}, u_{1}\right)+\frac{l_{1}}{V\left(\theta_{1}\right)}+T\left(\theta_{1}, \theta_{2}, u_{2}\right)+\frac{l_{2}}{V\left(\theta_{2}\right)}+T\left(\theta_{2}, \theta_{t}, u_{3}\right) .
$$

It must, however, be ensured that the path's total displacement is equal to $\left(x_{t}, y_{t}\right)$ so that the path ends up at the appropriate final configuration. Hence, the sum of the displacements achieved by each of the path's segments should equate $\left(x_{t}, y_{t}\right)$. This is equivalent to requiring that $c_{1}(\boldsymbol{\theta}, \boldsymbol{l}, \boldsymbol{u})=0$ and $c_{2}(\boldsymbol{\theta}, \boldsymbol{l}, \boldsymbol{u})=0$, where the constraint functions $c_{1}$ and $c_{2}$ are defined as:

$$
\begin{aligned}
& c_{1}(\boldsymbol{\theta}, \boldsymbol{l}, \boldsymbol{u})=P_{x}\left(\theta_{s}, \theta_{1}, u_{1}\right)+l_{1} \cos \theta_{1}+P_{x}\left(\theta_{1}, \theta_{2}, u_{2}\right)+l_{2} \cos \theta_{2}+P_{x}\left(\theta_{2}, \theta_{t}, u_{3}\right)-x_{t}, \\
& c_{2}(\boldsymbol{\theta}, \boldsymbol{l}, \boldsymbol{u})=P_{y}\left(\theta_{s}, \theta_{1}, u_{1}\right)+l_{1} \sin \theta_{1}+P_{y}\left(\theta_{1}, \theta_{2}, u_{2}\right)+l_{2} \sin \theta_{2}+P_{y}\left(\theta_{2}, \theta_{t}, u_{3}\right)-y_{t} .
\end{aligned}
$$

Additionally, the lengths of the straight line segments cannot, by definition, be negative, and it is required that $c_{3}(\boldsymbol{z}) \geq 0$ and $c_{4}(\boldsymbol{z}) \geq 0$, where $c_{3}(\boldsymbol{z})=l_{1}, c_{4}(\boldsymbol{z})=l_{2}$.

Finally, recall that the functions $T, P_{x}$ and $P_{y}$ were defined to make sense for $u \in\{-1,+1\}$. To guarantee that $u_{1}, u_{2}$ and $u_{3}$ do indeed belong to the latter set, it is imposed that the following three constraints be verified: $c_{5}(\boldsymbol{z})=0, c_{6}(\boldsymbol{z})=0$ and $c_{7}(\boldsymbol{z})=0$, where: $c_{5}(\boldsymbol{z})=u_{1}^{2}-1, c_{6}(\boldsymbol{z})=u_{2}^{2}-1, c_{7}(\boldsymbol{z})=u_{3}^{2}-1$.

Putting together all the above functions, the mathematical formulation of the problem can now be written:

$$
\begin{array}{rl}
\min _{\boldsymbol{z}} & f(\boldsymbol{z}), \\
\text { subject to } & c_{i}(\boldsymbol{z})=0, \quad i \in \mathcal{E} \\
& c_{i}(\boldsymbol{z}) \geq 0, \quad i \in \mathcal{I}
\end{array}
$$

where $\mathcal{E}=\{1,2,5,6,7\}$ is the set of equality constraints and $\mathcal{I}=\{3,4\}$ the set of inequality constraints. 
The necessary first and second order conditions that an optimal solution must satisfy can now be derived Nocedal and Wright, 2006. Once these conditions have been derived for the general case, they will be specified for the particular cases where it is known that the optimal solution is a $\mathcal{S C C}$ or $\mathcal{S C S}$ path. These con-

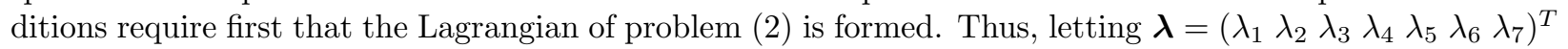
be the vector of Lagrangian multipliers, the Lagrangian reads:

$$
\mathcal{L}(\boldsymbol{z}, \boldsymbol{\lambda})=f(\boldsymbol{z})-\sum_{i \in \mathcal{E} \cup \mathcal{I}} \lambda_{i} c_{i}(\boldsymbol{z})
$$

\subsubsection{First order optimality conditions}

The first order conditions, or Karush-Kuhn-Tucker (KKT) conditions, state that in order for a vector $\left(\boldsymbol{z}^{*}, \boldsymbol{\lambda}^{*}\right)$ to be a solution to problem (2), it is necessary that $\nabla \mathcal{L}\left(\boldsymbol{z}^{*}, \boldsymbol{\lambda}^{*}\right)=0$. Of particular interest are the derivatives of $\mathcal{L}$ with respect to $\boldsymbol{\theta}$ and $\boldsymbol{l}$ at a candidate solution, which are computed here-under.

Consider the partial derivative of $\mathcal{L}$ with respect to $l_{1}$ :

$$
\frac{\partial \mathcal{L}}{\partial l_{1}}(\boldsymbol{z}, \boldsymbol{\lambda})=\frac{\partial f}{\partial l_{1}}(\boldsymbol{z})-\sum_{i \in \mathcal{E} \cup \mathcal{I}} \lambda_{i} \frac{\partial c_{i}}{\partial l_{1}}(\boldsymbol{z})=\frac{1}{V\left(\theta_{1}\right)}-\lambda_{1} \cos \theta_{1}-\lambda_{2} \sin \theta_{1}-\lambda_{3}=\frac{1}{V\left(\theta_{1}\right)}-\alpha \cos \left(\theta_{1}-\phi\right)-\lambda_{3},
$$

where $\alpha$ and $\phi$ are defined such that $\left(\lambda_{1}, \lambda_{2}\right)=(\alpha \cos \phi, \alpha \sin \phi)$. Similarly:

$$
\frac{\partial \mathcal{L}}{\partial l_{2}}(\boldsymbol{z}, \boldsymbol{\lambda})=\frac{1}{V\left(\theta_{2}\right)}-\alpha \cos \left(\theta_{2}-\phi\right)-\lambda_{4}
$$

Applying the KKT conditions and thus setting the two above equalities to zero, a candidate solution to the problem must satisfy:

$$
\begin{aligned}
& \frac{1}{V\left(\theta_{1}^{*}\right)}-\alpha^{*} \cos \left(\theta_{1}^{*}-\phi^{*}\right)=\lambda_{3}^{*}, \\
& \frac{1}{V\left(\theta_{2}^{*}\right)}-\alpha^{*} \cos \left(\theta_{2}^{*}-\phi^{*}\right)=\lambda_{4}^{*} .
\end{aligned}
$$

Consider now the partial derivative of $\mathcal{L}$ with respect to $\theta_{1}$ :

$$
\begin{aligned}
\frac{\partial \mathcal{L}}{\partial \theta_{1}}(\boldsymbol{z}, \boldsymbol{\lambda}) & =\frac{\partial f}{\partial \theta_{1}}(\boldsymbol{z})-\sum_{i \in \mathcal{E} \cup \mathcal{I}} \lambda_{i} \frac{\partial c_{i}}{\partial \theta_{1}}(\boldsymbol{z}) \\
& =\left(u_{1}-u_{2}\right) R\left(\theta_{1}\right)\left[\frac{1}{V\left(\theta_{1}\right)}-\alpha \cos \left(\theta_{1}-\phi\right)\right]-l_{1}\left[\frac{V^{\prime}\left(\theta_{1}\right)}{V\left(\theta_{1}\right)^{2}}-\alpha \sin \left(\theta_{1}-\phi\right)\right] .
\end{aligned}
$$

Similarly:

$$
\frac{\partial \mathcal{L}}{\partial \theta_{2}}(\boldsymbol{z}, \boldsymbol{\lambda})=\left(u_{2}-u_{3}\right) R\left(\theta_{2}\right)\left[\frac{1}{V\left(\theta_{2}\right)}-\alpha \cos \left(\theta_{2}-\phi\right)\right]-l_{2}\left[\frac{V^{\prime}\left(\theta_{2}\right)}{V\left(\theta_{2}\right)^{2}}-\alpha \sin \left(\theta_{2}-\phi\right)\right] .
$$

The KKT conditions are now applied and the previous two equalities are set to zero, while also making use of the conditions (3) and (4) derived above. This leads to the following two new conditions:

$$
\begin{aligned}
& l_{1}^{*}\left[\frac{V^{\prime}\left(\theta_{1}^{*}\right)}{V\left(\theta_{1}^{*}\right)^{2}}-\alpha^{*} \sin \left(\theta_{1}^{*}-\phi^{*}\right)\right]=\lambda_{3}^{*}\left(u_{1}^{*}-u_{2}^{*}\right) R\left(\theta_{1}^{*}\right), \\
& l_{2}^{*}\left[\frac{V^{\prime}\left(\theta_{2}^{*}\right)}{V\left(\theta_{2}^{*}\right)^{2}}-\alpha^{*} \sin \left(\theta_{2}^{*}-\phi^{*}\right)\right]=\lambda_{4}^{*}\left(u_{2}^{*}-u_{3}^{*}\right) R\left(\theta_{2}^{*}\right) .
\end{aligned}
$$

The conditions (3), (4), (5) and (6), while not in a particularly interesting form here, will turn out to have a deeper meaning in Sections 2.2 and 2.3. when further assumptions are made on the type of optimal path that solves problem (2). Having derived the needed first order conditions, second order necessary conditions are now considered in order to further narrow the family of candidate optimal paths. 


\subsubsection{Second order optimality conditions}

Consider a point $\left(\boldsymbol{z}^{*}, \boldsymbol{\lambda}^{*}\right)$ satisfying the KKT conditions (and hence conditions (3), (4), (5) and (6)). The second order conditions revolve around the Hessian $\nabla_{\boldsymbol{z} z} \mathcal{L}\left(\boldsymbol{z}^{*}, \boldsymbol{\lambda}^{*}\right)$ of the Lagrangian with respect to $\boldsymbol{z}$ at this candidate point $\left(\boldsymbol{z}^{*}, \boldsymbol{\lambda}^{*}\right)$. The second order derivative of the Lagrangian with respect to $\boldsymbol{z}$ need be computed.

The second derivatives of $\mathcal{L}$ at $\left(\boldsymbol{z}^{*}, \boldsymbol{\lambda}^{*}\right)$ are derived starting from the first derivatives computed in Section 2.1.3. To shorten notations, define the sets $I$ and $K$ as $I=\{1,2\}$ and $K=\{1,2,3\}$.

A number of second derivatives are null:

$$
\begin{aligned}
& \frac{\partial^{2} \mathcal{L}}{\partial l_{i} \partial \theta_{j}}(\boldsymbol{z}, \boldsymbol{\lambda})=0, \forall i \neq j \in I, \quad \frac{\partial^{2} \mathcal{L}}{\partial l_{i} \partial u_{k}}(\boldsymbol{z}, \boldsymbol{\lambda})=0, \forall i \in I, \forall k \in K, \quad \frac{\partial^{2} \mathcal{L}}{\partial l_{i} \partial l_{j}}(\boldsymbol{z}, \boldsymbol{\lambda})=0, \forall i, j \in I, \\
& \frac{\partial^{2} \mathcal{L}}{\partial \theta_{1} \partial \theta_{2}}(\boldsymbol{z}, \boldsymbol{\lambda})=0, \quad \frac{\partial^{2} \mathcal{L}}{\partial \theta_{1} \partial u_{3}}(\boldsymbol{z}, \boldsymbol{\lambda})=0, \quad \frac{\partial^{2} \mathcal{L}}{\partial \theta_{2} \partial u_{1}}(\boldsymbol{z}, \boldsymbol{\lambda})=0 .
\end{aligned}
$$

$\mathcal{L}$ is quadratic with respect to $u_{k}, k \in K$. Its second order derivatives with respect to $u_{k}, k \in K$ are thus readily found:

$$
\begin{gathered}
\frac{\partial^{2} \mathcal{L}}{\partial u_{k}^{2}}(\boldsymbol{z}, \boldsymbol{\lambda})=-2 \lambda_{k+4}, \forall k \in K . \\
\frac{\partial^{2} \mathcal{L}}{\partial l_{1} \partial \theta_{1}}(\boldsymbol{z}, \boldsymbol{\lambda})=-\frac{V^{\prime}\left(\theta_{1}\right)}{V\left(\theta_{1}\right)^{2}}+\alpha \sin \left(\theta_{1}-\phi\right) \text { and likewise for } \frac{\partial^{2} \mathcal{L}}{\partial l_{2} \partial \theta_{2}}(\boldsymbol{z}, \boldsymbol{\lambda}), \text { yielding: } \\
\frac{\partial^{2} \mathcal{L}}{\partial l_{1} \partial \theta_{1}}\left(\boldsymbol{z}^{*}, \boldsymbol{\lambda}^{*}\right)=-\frac{V^{\prime}\left(\theta_{1}^{*}\right)}{V\left(\theta_{1}^{*}\right)^{2}}+\alpha^{*} \sin \left(\theta_{1}^{*}-\phi^{*}\right), \\
\frac{\partial^{2} \mathcal{L}}{\partial l_{2} \partial \theta_{2}}\left(\boldsymbol{z}^{*}, \boldsymbol{\lambda}^{*}\right)=-\frac{V^{\prime}\left(\theta_{2}^{*}\right)}{V\left(\theta_{2}^{*}\right)^{2}}+\alpha^{*} \sin \left(\theta_{2}^{*}-\phi^{*}\right) . \\
\frac{\partial^{2} \mathcal{L}}{\partial \theta_{1} \partial u_{1}}(\boldsymbol{z}, \boldsymbol{\lambda})=R\left(\theta_{1}\right)\left[\frac{1}{V\left(\theta_{1}\right)}-\alpha \cos \left(\theta_{1}-\phi\right)\right], \quad \frac{\partial^{2} \mathcal{L}}{\partial \theta_{1} \partial u_{2}}(\boldsymbol{z}, \boldsymbol{\lambda})=-R\left(\theta_{1}\right)\left[\frac{1}{V\left(\theta_{1}\right)}-\alpha \cos \left(\theta_{1}-\phi\right)\right], \\
\frac{\partial^{2} \mathcal{L}}{\partial \theta_{2} \partial u_{2}}(\boldsymbol{z}, \boldsymbol{\lambda})=R\left(\theta_{2}\right)\left[\frac{1}{V\left(\theta_{2}\right)}-\alpha \cos \left(\theta_{2}-\phi\right)\right], \quad \frac{\partial^{2} \mathcal{L}}{\partial \theta_{2} \partial u_{3}}(\boldsymbol{z}, \boldsymbol{\lambda})=-R\left(\theta_{2}\right)\left[\frac{1}{V\left(\theta_{2}\right)}-\alpha \cos \left(\theta_{2}-\phi\right)\right],
\end{gathered}
$$

which using (3) and (4) gives:

$$
\begin{aligned}
\frac{\partial^{2} \mathcal{L}}{\partial \theta_{1} \partial u_{1}}\left(\boldsymbol{z}^{*}, \boldsymbol{\lambda}^{*}\right) & =R\left(\theta_{1}^{*}\right) \lambda_{3}^{*}, & \frac{\partial^{2} \mathcal{L}}{\partial \theta_{1} \partial u_{2}}\left(\boldsymbol{z}^{*}, \boldsymbol{\lambda}^{*}\right) & =-R\left(\theta_{1}^{*}\right) \lambda_{3}^{*}, \\
\frac{\partial^{2} \mathcal{L}}{\partial \theta_{2} \partial u_{2}}\left(\boldsymbol{z}^{*}, \boldsymbol{\lambda}^{*}\right) & =R\left(\theta_{2}\right) \lambda_{4}^{*}, & \frac{\partial^{2} \mathcal{L}}{\partial \theta_{2} \partial u_{3}}\left(\boldsymbol{z}^{*}, \boldsymbol{\lambda}^{*}\right) & =-R\left(\theta_{2}\right) \lambda_{4}^{*} .
\end{aligned}
$$

The last set of second order derivatives to be derived, and the ones that turn out to be the more informative, are $\frac{\partial^{2} \mathcal{L}}{\partial \theta_{i}^{2}}\left(\boldsymbol{z}^{*}, \boldsymbol{\lambda}^{*}\right), i \in I$. Consider for instance the case $i=1$ :

$$
\begin{aligned}
\frac{\partial^{2} \mathcal{L}}{\partial \theta_{1}^{2}}(\boldsymbol{z}, \boldsymbol{\lambda})=\left(u_{1}-u_{2}\right)[ & \left.R^{\prime}\left(\theta_{1}\right)\left(\frac{1}{V\left(\theta_{1}\right)}-\alpha \cos \left(\theta_{1}-\phi\right)\right)+R\left(\theta_{1}\right)\left(-\frac{V^{\prime}\left(\theta_{1}\right)}{V\left(\theta_{1}\right)^{2}}+\alpha \sin \left(\theta_{1}-\phi\right)\right)\right] \\
& -l_{1}\left[\frac{V^{\prime \prime}\left(\theta_{1}\right) V\left(\theta_{1}\right)-2 V^{\prime}\left(\theta_{1}\right)^{2}}{V\left(\theta_{1}\right)^{3}}-\alpha \cos \left(\theta_{1}-\phi\right)\right],
\end{aligned}
$$

which, using (3), yields:

$$
\frac{\partial^{2} \mathcal{L}}{\partial \theta_{1}^{2}}\left(\boldsymbol{z}^{*}, \boldsymbol{\lambda}^{*}\right)=\lambda_{3}^{*}\left[\left(u_{1}^{*}-u_{2}^{*}\right) R^{\prime}\left(\theta_{1}^{*}\right)-\frac{l_{1}^{*}}{V\left(\theta_{1}^{*}\right)^{3}}\right]+\left(u_{1}^{*}-u_{2}^{*}\right) R\left(\theta_{1}^{*}\right)\left[-\frac{V^{\prime}\left(\theta_{1}^{*}\right)}{V\left(\theta_{1}^{*}\right)^{2}}+\alpha^{*} \sin \left(\theta_{1}^{*}-\phi^{*}\right)\right]+\frac{l_{1}^{*}}{V\left(\theta_{1}^{*}\right)^{3}} \gamma\left(\theta_{1}^{*}\right),
$$

and likewise:

$$
\frac{\partial^{2} \mathcal{L}}{\partial \theta_{2}^{2}}\left(\boldsymbol{z}^{*}, \boldsymbol{\lambda}^{*}\right)=\lambda_{4}^{*}\left[\left(u_{2}^{*}-u_{3}^{*}\right) R^{\prime}\left(\theta_{2}^{*}\right)-\frac{l_{2}^{*}}{V\left(\theta_{2}^{*}\right)^{3}}\right]+\left(u_{2}^{*}-u_{3}^{*}\right) R\left(\theta_{2}^{*}\right)\left[-\frac{V^{\prime}\left(\theta_{2}^{*}\right)}{V\left(\theta_{2}^{*}\right)^{2}}+\alpha^{*} \sin \left(\theta_{2}^{*}-\phi^{*}\right)\right]+\frac{l_{2}^{*}}{V\left(\theta_{2}^{*}\right)^{3}} \gamma\left(\theta_{2}^{*}\right),
$$


where the function $\gamma$ is defined as: $\gamma(\theta)=V(\theta)^{2}-V^{\prime \prime}(\theta) V(\theta)+2 V^{\prime}(\theta)^{2}$.

While never formally stated, it is implied by the analysis above that $V$ and $R$ are required to be twice differentiable and differentiable respectively.

The choice of defining this function $\gamma$ is neither arbitrary nor merely to avoid cumbersome notations. It is actually interesting to observe that $\gamma(\theta)$ is the expression that appears in the numerator of the signed curvature of the speed function $V$. Indeed, for a curve defined in polar coordinates as $r(\theta)$, its signed curvature $\kappa(\theta)$ is expressed as $\kappa(\theta)=\frac{r(\theta)^{2}-r^{\prime \prime}(\theta) r(\theta)+2 r^{\prime}(\theta)^{2}}{\left(r^{2}+r^{\prime 2}\right)^{3 / 2}}$. The implication of this observation is that the sign of $\gamma(\theta)$ informs on the behavior of the curve at $\theta$. At a given $\theta$, the polar plot of $V$ is convex if $\gamma(\theta) \geq 0$, and concave if $\gamma(\theta) \leq 0$. This property will be used in Sections 2.2 and 2.3 to express geometrical properties satisfied by the speed polar plot at $\theta_{1}^{*}$ and $\theta_{2}^{*}$.

The above second derivatives yield the elements of the Hessian $\nabla_{\boldsymbol{z} z} \mathcal{L}$ of $\mathcal{L}$. Recall that given $\left(\boldsymbol{z}^{*}, \boldsymbol{\lambda}^{*}\right)$ satisfying the KKT conditions, the critical cone $\mathcal{C}\left(\boldsymbol{z}^{*}, \boldsymbol{\lambda}^{*}\right)$ is defined as:

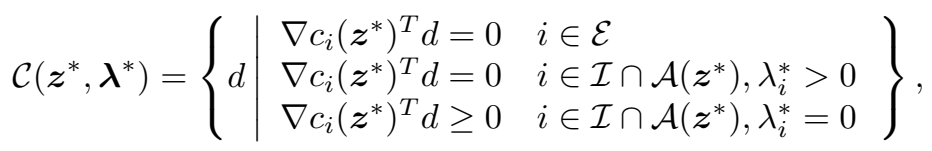

where $\mathcal{A}\left(\boldsymbol{z}^{*}\right)$ is the set of active constraints at $\boldsymbol{z}^{*}$ Nocedal and Wright, 2006, ch.12]. A second order necessary condition then states that if $\left(\boldsymbol{z}^{*}, \boldsymbol{\lambda}^{*}\right)$ satisfies the KKT conditions and the Linear Independence Constraint Qualification (LICQ) hold at $\boldsymbol{z}^{*}$, then: $\boldsymbol{w}^{T} \nabla_{\boldsymbol{z} \boldsymbol{z}} \mathcal{L}\left(\boldsymbol{z}^{*}, \boldsymbol{\lambda}^{*}\right) \boldsymbol{w} \geq 0, \quad \forall \boldsymbol{w} \in \mathcal{C}\left(\boldsymbol{z}^{*}, \boldsymbol{\lambda}^{*}\right)$. The LICQ corresponds to the independence of the gradients of the active constraints at $\boldsymbol{z}^{*}$.

\section{$2.2 \quad$ Necessary conditions for an optimal $\mathcal{S C S}$ path}

In Section 2.1, some expressions and relations that an optimal path must satisfy were derived, which do not seem very insightful in their raw form. In this section and the next, some particular candidate solutions are analyzed in more detail.

It is supposed throughout Section 2.2 that there exists a solution to problem (2) that is a $\mathcal{S C S}$ path. Consequently, assume the following: $l_{1}^{*}>0, l_{2}^{*}>0$ and $\theta_{1}^{*} \neq \theta_{2}^{*}$.

\subsubsection{First order necessary conditions for an optimal $\mathcal{S C S}$ path}

This additional information can be used by applying the complementary slackness property required by the KKT conditions. In other words, for any $i \in \mathcal{E} \cup \mathcal{I}$, it must be that $\lambda_{i}^{*} c_{i}\left(\boldsymbol{z}^{*}\right)=0$. This is particularly interesting for $i=3$ and $i=4$ for it yields that necessarily, $\lambda_{3}^{*} l_{1}^{*}=\lambda_{4}^{*} l_{2}^{*}=0$. Since it is assumed that $l_{1}^{*}, l_{2}^{*}>0$, it follows that:

$$
\lambda_{3}^{*}=0, \quad \lambda_{4}^{*}=0 .
$$

These two equalities have interesting consequences, for when plugged back into (3) and (4) they yield:

$$
\begin{aligned}
& \frac{1}{\alpha^{*}}=V\left(\theta_{1}^{*}\right) \cos \left(\theta_{1}^{*}-\phi^{*}\right), \\
& \frac{1}{\alpha^{*}}=V\left(\theta_{2}^{*}\right) \cos \left(\theta_{2}^{*}-\phi^{*}\right) .
\end{aligned}
$$

Equations (8) and (9) have a geometrical interpretation that is displayed in Figure 3 . Indeed, $\phi^{*}$ can be interpreted as a direction along which the projections of the speed vectors with headings $\theta_{1}^{*}$ and $\theta_{2}^{*}$ are the same and equal to $1 / \alpha^{*}$. Note that these equations are well defined for it follows from the complementary slackness property that $\lambda_{1}^{*} \neq 0$ and $\lambda_{2}^{*} \neq 0$, and consequently $\alpha^{*} \neq 0$. It can further be observed that equations (8) and (9) imply that it cannot be that $\theta_{1}^{*}=\theta_{2}^{*} \bmod \pi$, which is sensible since it would otherwise mean - if the straight-line segments are of non-zero length - that one would travel forward and backward along parallel straight lines, which is clearly sub-optimal.

Equations (8) and (9) can further be used and substituting them into equations (5) and (6) yields:

$$
\begin{aligned}
& V^{\prime}\left(\theta_{1}^{*}\right)=V\left(\theta_{1}^{*}\right) \tan \left(\theta_{1}^{*}-\phi^{*}\right), \\
& V^{\prime}\left(\theta_{2}^{*}\right)=V\left(\theta_{2}^{*}\right) \tan \left(\theta_{2}^{*}-\phi^{*}\right) .
\end{aligned}
$$




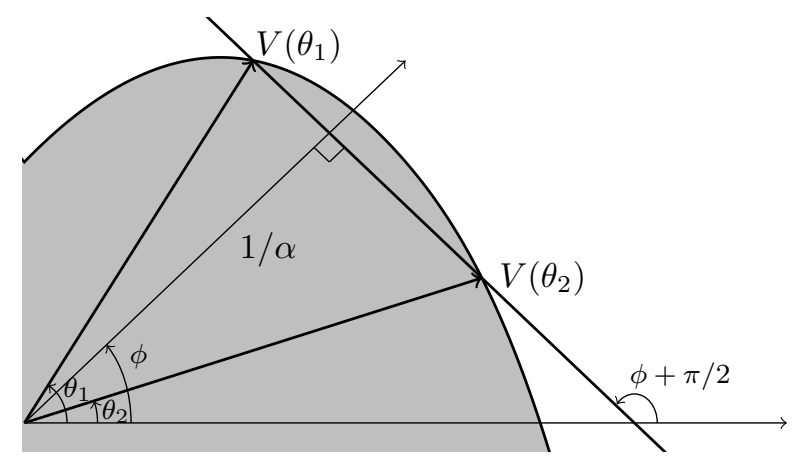

Figure 3: Geometrical interpretation of Equations (8) and (9).

There is additional geometrical insight to be seen in these two equations by using the fact that the relationship between $V^{\prime}$ and the tangent to the graph of $V$ is $V^{\prime}(\theta)=V(\theta) \cot (\zeta(\theta))$, where $\zeta(\theta)$ is the angle between the tangent and radial lines of the polar plot of $V(\theta)$ (see for instance Youse 1978, p.676]). Substituting into the above equations yields:

$$
\begin{aligned}
& \theta_{1}^{*}+\zeta\left(\theta_{1}^{*}\right)=\phi^{*}+\frac{\pi}{2} \quad \bmod \pi \\
& \theta_{2}^{*}+\zeta\left(\theta_{2}^{*}\right)=\phi^{*}+\frac{\pi}{2} \quad \bmod \pi
\end{aligned}
$$

Equations 12 and 13 have a strong geometrical significance since they imply that the tangents to the speed polar plot at $\theta_{1}^{*}$ and $\theta_{2}^{*}$ are parallel to each other and to a line with an angular direction of $\phi^{*}+\pi / 2$. However, $\phi^{*}+\pi / 2$ is by construction the angular direction of the line passing through the points with polar coordinates $\left(\theta_{1}^{*}, V\left(\theta_{1}^{*}\right)\right)$ and $\left(\theta_{2}^{*}, V\left(\theta_{2}^{*}\right)\right)$. The following proposition, which is illustrated in Figure 4 , has thus been proved:

Proposition 1. Let $V: S^{1} \rightarrow \mathbb{R}^{+}$be a positive and differentiable speed function and $R: S^{1} \rightarrow \mathbb{R}^{+}$be a positive minimum-turning radius function. Suppose an optimal path from an initial position $\left(x_{s}, y_{s}, \theta_{s}\right)$ to a final position $\left(x_{t}, y_{t}, \theta_{t}\right)$ contains a non-degenerate sub-path of the form $\mathcal{S C S}$, where the straight-line segments have headings $\theta_{1}$ and $\theta_{2}$, respectively. Then, the line connecting the points $\left(\theta_{1}, V\left(\theta_{1}\right)\right)$ and $\left(\theta_{2}, V\left(\theta_{2}\right)\right)$ in polar coordinates coincides with the tangents to the speed polar plot at those points (see Figure 4 ).

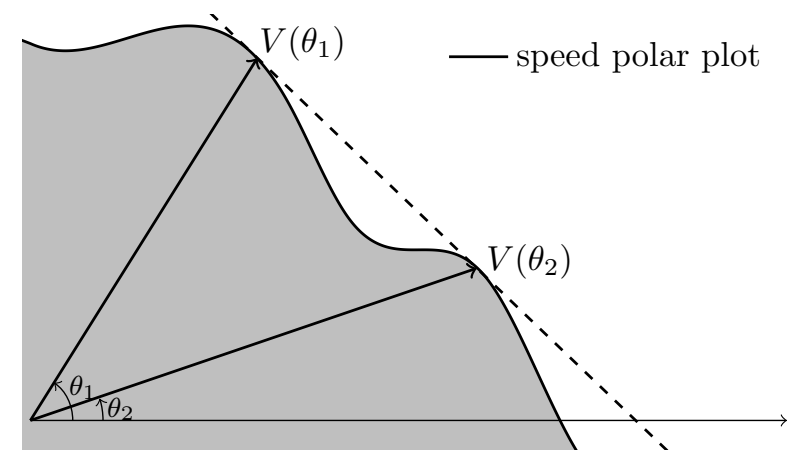

Figure 4: Illustration of Proposition 1

\subsubsection{Second order necessary conditions for an optimal $\mathcal{S C S}$ path}

Proposition 1 states that the line passing through $\left(\theta_{1}, V\left(\theta_{1}\right)\right)$ and $\left(\theta_{2}, V\left(\theta_{2}\right)\right)$ (in polar coordinates), where $\theta_{1}$ and $\theta_{2}$ are the straight line segments' headings, is a bitangent to the speed polar plot. This characterization 
still leaves open many combinations as can be seen on Figure 5. Bitangents or double-tangents have been studied in the mathematical literature, notably by Fabricius-Bjerre [1962]. He distinguishes between interior and exterior double tangents depending on whether "the convex arcs in the neighborhood of the points of contact $R_{1}$ and $R_{2}$ are on the same side of $r$ or on opposite sides" - where $r$ denotes the double tangent. In the case of an exterior bitangent, where both contact points are on the same side of the tangent, two cases can be distinguished: 1) convex bitangents whose points of contact are located on convex segments of the speed polar plot (such as bitangent 1 on Figure 5), and 2) concave bitangents whose points of contact are located on concave segments of the speed polar plot (such as bitangent 2 on Figure 5).

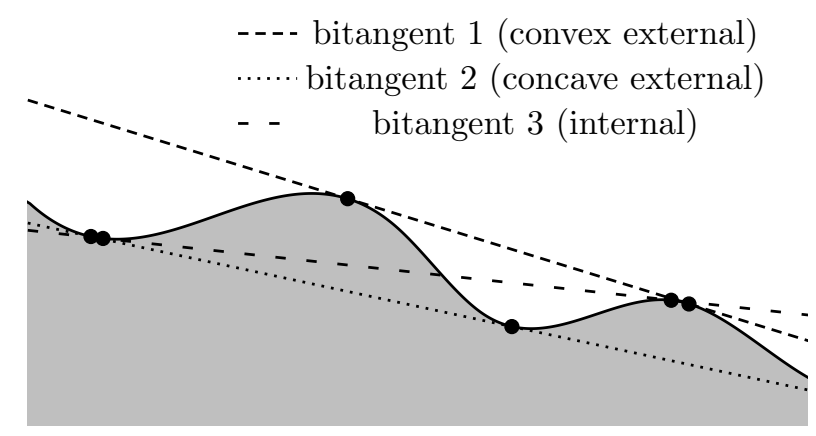

Figure 5: Examples of bitangents to the speed polar plot satisfying Proposition 1 .

In laying out the initial calculations towards second order conditions in Section 2.1.4, it was observed that a term related to the curvature of the speed polar plot arose naturally, hinting at a possible role of the curvature of the speed polar plot in the optimality, or lack thereof, of a candidate path. This is indeed the case, and second order conditions actually enable the classification of the bitangent as a convex bitangent as shown below.

Let $\left(\boldsymbol{z}^{*}, \boldsymbol{\lambda}^{*}\right)$ correspond to the optimal solution and thus satisfy the KKT conditions. Using equations (7)-11), and substituting these relations in the expressions of the second derivatives of $\mathcal{L}$ at $\left(\boldsymbol{z}^{*}, \boldsymbol{\lambda}^{*}\right)$ yields a Hessian matrix displaying a simple structure, as it is diagonal:

$$
\nabla_{\boldsymbol{z} \boldsymbol{z}} \mathcal{L}\left(\boldsymbol{z}^{*}, \boldsymbol{\lambda}^{*}\right)=\begin{array}{cccccccc}
\theta_{1} & \theta_{2} & l_{1} & l_{2} & u_{1} & u_{2} & u_{3} \\
& \theta_{1} \\
\theta_{2} & l_{1} \\
l_{2} & u_{1} \\
u_{2} \\
u_{3}
\end{array}
$$

Recall from Section 2.1.4 that given a vector $\boldsymbol{w}$ in the critical cone at $\left(\boldsymbol{z}^{*}, \boldsymbol{\lambda}^{*}\right)$, and provided the LICQ holds, a necessary condition for $\left(\boldsymbol{z}^{*}, \boldsymbol{\lambda}^{*}\right)$ to be optimal is that $\boldsymbol{w}^{T} \nabla_{\boldsymbol{z} \boldsymbol{z}} \mathcal{L}\left(\boldsymbol{z}^{*}, \boldsymbol{\lambda}^{*}\right) \boldsymbol{w} \geq 0$. It can be seen in particular that if vectors belonging to the critical cone of the form $\boldsymbol{w}_{1}=\left(\begin{array}{lllll}\star & 0 \star & 0 & 0 & 0\end{array}\right)^{T}$ and $\boldsymbol{w}_{2}=\left(\begin{array}{llllll}0 & \star & \star & 0 & 0 & 0\end{array}\right)^{T}$, where $\star$ denotes a non-null element, can be found, then $\boldsymbol{w}_{j}^{T} \nabla_{\boldsymbol{z}} \mathcal{L}\left(\boldsymbol{z}^{*}, \boldsymbol{\lambda}^{*}\right) \boldsymbol{w}_{j}$ will have the sign of $\gamma\left(\theta_{j}^{*}\right)(j=1,2)$, thus informing on the curvature of the speed polar plot at the optimal angles $\theta_{1}^{*}$ and $\theta_{2}^{*}$.

The first step is to check that the LICQ holds. Letting $A(\boldsymbol{z})$ be the matrix whose rows are the gradients 
of the constraints at $\boldsymbol{z}$ gives:

$$
\begin{aligned}
& \begin{array}{lllllll}
\theta_{1} & \theta_{2} & l_{1} & l_{2} & u_{1} & u_{2} & u_{3}
\end{array}
\end{aligned}
$$

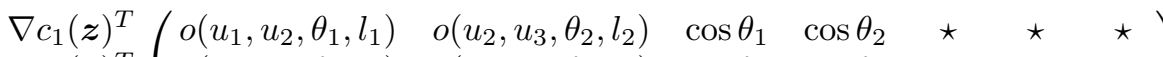

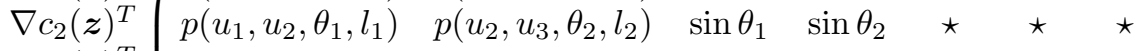

$$
\begin{aligned}
& A(\boldsymbol{z})=\begin{array}{c}
\nabla c_{3}(\boldsymbol{z})^{T} \\
\nabla c_{4}(\boldsymbol{z})^{T} \\
\nabla c_{5}(\boldsymbol{z})^{T} \\
\nabla c_{6}(\boldsymbol{z})^{T} \\
\nabla c_{7}(\boldsymbol{z})^{T}
\end{array}
\end{aligned}
$$

where $o\left(u, u^{\prime}, \theta, l\right)=\left(u-u^{\prime}\right) R(\theta) \cos \theta-l \sin \theta$ and $p\left(u, u^{\prime}, \theta, l\right)=\left(u-u^{\prime}\right) R(\theta) \sin \theta+l \cos \theta$.

It can be observed that $A\left(\boldsymbol{z}^{*}\right)$ has full rank and, consequently, the LICQ holds. Furthermore, it can be verified that the vectors $\boldsymbol{w}_{1}$ and $\boldsymbol{w}_{2}$ defined as:

$$
\begin{aligned}
& \boldsymbol{w}_{1}=\left(1,0,-\left[\left(u_{1}^{*}-u_{2}^{*}\right) R\left(\theta_{1}^{*}\right)+l_{1}^{*} \cot \left(\theta_{1}^{*}-\theta_{2}^{*}\right)\right], \frac{l_{1}^{*}}{\sin \left(\theta_{1}^{*}-\theta_{2}^{*}\right)}, 0,0,0\right)^{T}, \\
& \boldsymbol{w}_{2}=\left(0,1,-\frac{l_{2}^{*}}{\sin \left(\theta_{1}^{*}-\theta_{2}^{*}\right)}, \quad-\left[\left(u_{2}^{*}-u_{3}^{*}\right) R\left(\theta_{2}^{*}+l_{2}^{*} \cot \left(\theta_{1}^{*}-\theta_{2}^{*}\right)\right], 0,0,0\right)^{T},\right.
\end{aligned}
$$

belong to the critical cone $\mathcal{C}\left(\boldsymbol{z}^{*}, \boldsymbol{\lambda}^{*}\right)$. It follows that a necessary condition for $\left(\boldsymbol{z}^{*}, \boldsymbol{\lambda}^{*}\right)$ to be optimal is that the following two inequalities hold:

$$
\begin{aligned}
& \boldsymbol{w}_{1}^{T} \nabla_{\boldsymbol{z}} \mathcal{L}\left(\boldsymbol{z}^{*}, \boldsymbol{\lambda}^{*}\right) \boldsymbol{w}_{1} \geq 0 \quad \Longrightarrow \gamma\left(\theta_{1}^{*}\right) \geq 0, \\
& \boldsymbol{w}_{2}^{T} \nabla_{\boldsymbol{z} \boldsymbol{z}} \mathcal{L}\left(\boldsymbol{z}^{*}, \boldsymbol{\lambda}^{*}\right) \boldsymbol{w}_{2} \geq 0 \quad \Longrightarrow \gamma\left(\theta_{2}^{*}\right) \geq 0
\end{aligned}
$$

Hence, the speed polar plot is convex at both $\theta_{1}^{*}$ and $\theta_{2}^{*}$. The following proposition characterizes this result and can be seen as a generalization of Corollary 4.6 in Chang 2011.

Proposition 2. Let $V: S^{1} \rightarrow \mathbb{R}^{+}$be a positive and twice differentiable speed function and $R:[0,2 \pi] \rightarrow \mathbb{R}^{+}$ be a positive and differentiable minimum-turning radius function. Suppose an optimal path from an initial position $\left(x_{s}, y_{s}, \theta_{s}\right)$ to a final position $\left(x_{t}, y_{t}, \theta_{t}\right)$ contains a non-degenerate sub-path of the form $\mathcal{S C S}$, where the straight-line segments have distinct headings $\theta_{1}$ and $\theta_{2}$, respectively. Then the line passing through $\left(\theta_{1}, V\left(\theta_{1}\right)\right)$ and $\left(\theta_{2}, V\left(\theta_{2}\right)\right)$ (in polar coordinates) is a convex bitangent to the speed polar plot.

\subsection{Necessary condition for an optimal $\mathcal{S C C}$ path}

Proceed in a similar fashion for $\mathcal{S C C}$ paths, by applying first and second order necessary conditions for optimality, which yield results similar to those obtained for $\mathcal{S C S}$ paths.

It is assumed throughout Section 2.3 that there exists an optimal $\mathcal{S C C}$ path to problem (2). Without loss of generality, it can be assumed that the straight line segment has heading $\theta_{1}$ and that the turn switch happens with heading $\theta_{2}$. Then the following conditions hold: $l_{1}^{*}>0, l_{2}^{*}=0, \theta_{1}^{*} \neq \theta_{2}^{*}$, and $u_{2}^{*} \neq u_{3}^{*}$.

\subsubsection{First order necessary conditions for an optimal $\mathcal{S C C}$ path}

As in Section 2.2, the complementarity slackness condition immediately yields $\lambda_{3}^{*}=0$. Furthermore, since $l_{2}^{*}=0$, (6) implies that $\lambda_{4}^{*}\left(u_{2}^{*}-u_{3}^{*}\right) R\left(\theta_{2}^{*}\right)=0$. Since it is further assumed that $u_{2}^{*} \neq u_{3}^{*}$ and the minimumturning radius is positive, it follows that $\lambda_{4}^{*}=0$ as well. It thus follows, as in Section 2.2 , that:

$$
\begin{aligned}
\frac{1}{\alpha^{*}} & =V\left(\theta_{1}^{*}\right) \cos \left(\theta_{1}^{*}-\phi^{*}\right), \\
\frac{1}{\alpha^{*}} & =V\left(\theta_{2}^{*}\right) \cos \left(\theta_{2}^{*}-\phi^{*}\right) .
\end{aligned}
$$

Additionally, $\lambda_{3}^{*}=0$ implies through (5) that 10 still holds in the present case, i.e.,

$$
V^{\prime}\left(\theta_{1}^{*}\right)=V\left(\theta_{1}^{*}\right) \tan \left(\theta_{1}^{*}-\phi^{*}\right) .
$$


However, 11 does not hold here. Consequently, the geometrical interpretation of these equations is slightly different in the case of an optimal $\mathcal{S C C}$ path from that of an optimal $\mathcal{S C S}$. It can now only be stated that the line passing through $\left(\theta_{1}^{*}, V\left(\theta_{1}^{*}\right)\right)$ and $\left(\theta_{2}^{*}, V\left(\theta_{2}^{*}\right)\right)$ in polar coordinates coincides with the tangent to the speed polar plot at $\left(\theta_{1}^{*}, V\left(\theta_{1}^{*}\right)\right)$, but not necessarily with the tangent to the speed polar plot at $\left(\theta_{2}^{*}, V\left(\theta_{2}^{*}\right)\right)$ (see Figure 6).

Proposition 3. Let $V: S^{1} \rightarrow \mathbb{R}^{+}$be a positive and differentiable speed function and $R: S^{1} \rightarrow \mathbb{R}^{+}$be a positive minimum-turning radius function. Suppose an optimal path from an initial position $\left(x_{s}, y_{s}, \theta_{s}\right)$ to a final position $\left(x_{t}, y_{t}, \theta_{t}\right)$ contains a non-degenerate sub-path of the form $\mathcal{S C C}$ (resp. $\mathcal{C C S}$ ) where the straight-line segment has a heading $\theta_{1}$, and $\theta_{2}$ is the heading where the two consecutive sharpest turn curves join. Then, the line connecting the points $\left(\theta_{1}, V\left(\theta_{1}\right)\right)$ and $\left(\theta_{2}, V\left(\theta_{2}\right)\right)$ in polar coordinates coincides with the tangent to the speed polar plot at $\left(\theta_{1}, V\left(\theta_{1}\right)\right)$ (see Figure [6).

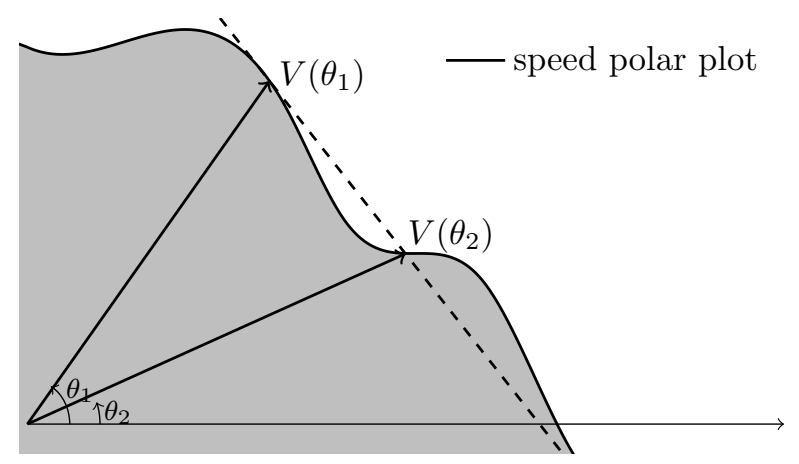

Figure 6: Illustration of Proposition 3.

It is also important to observe that while Proposition 3 gives information on the location of the straight line, it also tells something about where it is not. Since the tangent to the speed polar plot at $\left(\theta_{1}^{*}, V\left(\theta_{1}^{*}\right)\right)$ must intersect the speed polar point at a second point, it implies that no path containing a $\mathcal{S C C}$ path has a straight line with a heading $\theta_{1}^{*}$ such that $\theta_{1}^{*}$ is a bordering angle. It also means that any optimal path with a single straight line whose heading $\theta_{d}$ is a bordering angle is necessarily a Dubins-like path of the form $\mathcal{C S C}$.

\subsubsection{Second order necessary conditions for an optimal $\mathcal{S C C}$ path}

The derivation of second order necessary conditions for optimality of a $\mathcal{S C C}$ path closely resembles that of the second order necessary conditions for the optimality of a $\mathcal{S C S}$ path. Observe that a great number of relations and equalities are shared by both types of paths. The only differences are that, in the case of an optimal $\mathcal{S C C}$ path, one has $l_{2}^{*}=0$ instead of $l_{2}^{*}>0$ and that equation (11) no longer holds. The consequence of these two differences is that the Hessian matrix $\nabla_{\boldsymbol{z} \boldsymbol{z}} \mathcal{L}\left(\boldsymbol{z}^{*}, \boldsymbol{\lambda}^{*}\right)$, while still diagonal, has a slightly different form from the one displayed in (14). The only difference is in the term $\frac{\partial^{2} \mathcal{L}}{\partial \theta_{2}^{2}}$, which now reads $\left(u_{2}^{*}-u_{3}^{*}\right) R\left(\theta_{2}^{*}\right)\left(\tan \left(\theta_{2}^{*}-\phi^{*}\right)-\cot \zeta\left(\theta_{2}^{*}\right)\right)$ instead of $\frac{l_{2}^{*}}{V\left(\theta_{2}^{*}\right)^{3}} \gamma\left(\theta_{2}^{*}\right)$.

The matrix $A\left(\boldsymbol{z}^{*}\right)$ has full rank here as well, and consequently the LICQ holds. It is easily checked that the vector $\boldsymbol{w}_{1}$ defined in 15 belongs to the critical cone $\mathcal{C}\left(\boldsymbol{z}^{*}, \boldsymbol{\lambda}^{*}\right)$. Thus, a necessary condition for $\left(\boldsymbol{z}^{*}, \boldsymbol{\lambda}^{*}\right)$ to be optimal is for the following equality to hold:

$$
\boldsymbol{w}_{1}^{T} \nabla_{\boldsymbol{z} \boldsymbol{z}} \mathcal{L}\left(\boldsymbol{z}^{*}, \boldsymbol{\lambda}^{*}\right) \boldsymbol{w}_{1} \geq 0 \quad \Longrightarrow \gamma\left(\theta_{1}^{*}\right) \geq 0 .
$$

Hence, the speed polar plot is convex at $\theta_{1}^{*}$. However, due to the change in the structure of the Hessian, the same conclusion cannot be reached for $\theta_{2}^{*}$. It was also observed in the previous section that the heading $\theta_{1}^{*}$ of the straight line could not be a bordering angle. Combining these observations yields the following proposition.

Proposition 4. Consider an optimal SCC path whose straight line segment's heading is $\theta_{1}$. Then $\theta_{1}$ is a non-bordering convex angle. 


\section{Fastest-path algorithm for differentiable speed functions}

In Section 2, additional necessary properties for $\mathcal{S C C}$ and $\mathcal{S C S}$ paths to be optimal were derived. These additional characteristics of optimal paths narrow down the structure of paths that need to be considered for optimality. One way to implement an algorithm that determines the fastest path between an initial and a final configurations is thus to consider the candidate paths belonging to each of the three types and select the best. This idea is the one behind most implementations of algorithms solving the Dubins car problem, wherein all the possible paths are evaluated. This approach is valid so long as there is only a finite set of candidate paths to choose from. It is already known that there are indeed finitely many - at most six - Dubins-like paths, which have a structure of the form $\mathcal{C C C}$ or $\mathcal{C S C}$ (or a degeneracy of these), and the fastest of such paths can be derived using Algorithm 1 from Dolinskaya and Maggiar 2012]. However, there is increased complexity since, in addition to the Dubins-like paths, one needs to consider $\mathcal{S C C}$ and $\mathcal{S C S}$ paths in the general case. Propositions 2 and 4 reduce the sets of paths to which optimal $\mathcal{S C C}$ and $\mathcal{S C S}$ paths belong. In particular, it is known from Proposition 2 that the headings $\theta_{1}$ and $\theta_{2}$ of the straight lines in an optimal $\mathcal{S C S}$ path must be such that the line passing through $\left(\theta_{1}, V\left(\theta_{1}\right)\right)$ and $\left(\theta_{2}, V\left(\theta_{2}\right)\right.$ ) (in polar coordinates) is a bitangent (or double tangent) to the speed polar plot. Making additional mild and reasonable assumptions on the speed polar plot, it can be ensured that there are only finitely many pairs of headings satisfying this property and, consequently, only finitely many candidate $\mathcal{S C S}$ paths. This is a desirable statement in order to implement the algorithm since it makes it possible to enumerate all such paths.

Unfortunately, the same does not hold for $\mathcal{S C C}$ paths, since Proposition 3 only asserts that the line passing through $\left(\theta_{1}, V\left(\theta_{1}\right)\right)$ and $\left(\theta_{2}, V\left(\theta_{2}\right)\right)$ must coincide with the tangent to the speed polar plot at $\left(\theta_{1}, V\left(\theta_{1}\right)\right)$ (where $\theta_{1}$ is the heading of the straight line and $\theta_{2}$ is the heading where the two consecutive sharpest turn curves join). The sets of headings $\theta_{1}$ where a straight line of a $\mathcal{S C C}$ path may occur are possibly uncountable and correspond to the intervals of non-bordering convex headings. This prevents applying an enumeration method on the $\mathcal{S C C}$ paths, and an alternative assumption or approach to overcome this difficulty must be found. In the similar problem faced by Chang 2011, the author resorted to using piecewise constant speed polar plots, which were a reasonable fit to the motivation in his work. A general algorithm that computes a fastest path between an initial and a final configurations of a vehicle with a twice-differentiable positive speed function and a differentiable minimum-turning radius function is presented. The only assumption that is made in this section is that the speed polar plot has a finite number of bitangents. This is a very mild assumption that is always satisfied for practical purposes, and it enables the enumeration all the candidate $\mathcal{S C S}$ paths.

In the following sections each type of path (Dubins-like, $\mathcal{S C S}$ and $\mathcal{S C C}$ ) is considered and discussed: first briefly restating the algorithm for convex speed polar plots presented in Dolinskaya and Maggiar 2012 and then analyzing in more detail $\mathcal{S C S}$ and $\mathcal{S C C}$ paths.

\subsection{Construction of Dubins-like paths}

Algorithm 1 presented in Dolinskaya and Maggiar 2012 is an algorithm that solves the fastest-path problem for a convex speed polar plot, which also corresponds to solving for Dubins-like paths.

\subsection{Construction of $\mathcal{S C S}$ paths}

Consider a candidate path whose sharpest turn directions are given by $k_{i} \in\{L, R\}, i \in\{1,2,3\}$ in order of appearance, and whose straight line segments have headings $\theta_{1}$ and $\theta_{2}$. This yields a path of the form $\mathcal{C}_{k_{1}}\left(\theta_{s}, \theta_{1}\right) \mathcal{S C}_{k_{2}}\left(\theta_{1}, \theta_{2}\right) \mathcal{S C}_{k_{3}}\left(\theta_{2}, \theta_{t}\right)$ where the only unknown parameters are the lengths $l_{1}$ and $l_{2}$ of the straight lines. Note that such a path is not necessarily feasible. To determine its feasibility and evaluate its travel time, the function SCStime, whose pseudo-code is displayed in Table 1 is used. The function takes $k_{i} \in$ $\{L, R\}, i \in\{1,2,3\}, \theta_{1}$ and $\theta_{2}$ as inputs and outputs the corresponding path-time $\tau$.

The parameters $l_{1}$ and $l_{2}$ are easily derived noting that the displacement achieved by the two straight lines added to the displacement of the three turns $\mathcal{C}_{k_{1}}\left(\theta_{s}, \theta_{1}\right), \mathcal{C}_{k_{2}}\left(\theta_{1}, \theta_{2}\right)$ and $\mathcal{C}_{k_{3}}\left(\theta_{2}, \theta_{t}\right)$ must equal $\left(x_{t}, y_{t}\right)$. Since the values of $\theta_{1}, \theta_{2}, k_{i}, i=1,2,3$ are fixed, one can compute the displacement of the three turns and simply find the values of $l_{1}$ and $l_{2}$ that cover the remaining displacement to reach $\left(x_{t}, y_{t}\right)$ (see Figure 7). Letting 


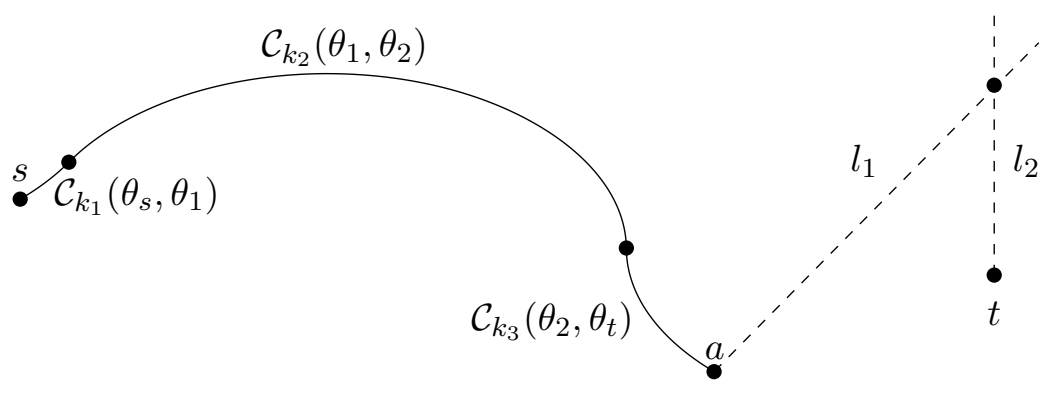

Figure 7: Construction of $\mathcal{S C S}$ paths

$\boldsymbol{a}=\left(x_{a}, y_{a}\right)$ be the total displacement achieved by the three turns, it follows that $a:=D\left(\mathcal{C}_{k_{1}}\left(\theta_{s}, \theta_{1}\right)\right)+$ $D\left(\mathcal{C}_{k_{2}}\left(\theta_{1}, \theta_{2}\right)\right)+D\left(\mathcal{C}_{k_{3}}\left(\theta_{2}, \theta_{t}\right)\right)$, whence the system solved by $l_{1}$ and $l_{2}$ reads:

$$
\left\{\begin{array}{ll}
l_{1} \cos \theta_{1}+l_{2} \cos \theta_{2} & =x_{t}-x_{a} \\
l_{1} \sin \theta_{1}+l_{2} \sin \theta_{2} & =y_{t}-y_{a}
\end{array},\right.
$$

the solution of which is:

$$
\begin{aligned}
& l_{1}=\frac{\left(x_{t}-x_{a}\right) \sin \theta_{2}-\left(y_{t}-y_{a}\right) \cos \theta_{2}}{\sin \left(\theta_{2}-\theta_{1}\right)}, \\
& l_{2}=\frac{\left(x_{t}-x_{a}\right) \sin \theta_{1}-\left(y_{t}-y_{a}\right) \cos \theta_{1}}{\sin \left(\theta_{1}-\theta_{2}\right)} .
\end{aligned}
$$

Note that these quantities are never ill-defined for it was observed in Section 2.2 that $\theta_{1}^{*} \neq \theta_{2}^{*} \bmod \pi$, preventing the denominator in the two above equations from being null. If either $l_{1}$ or $l_{2}$ is negative, then the candidate path is not feasible and its corresponding travel time is set to infinity, otherwise its travel time is obtained as $f(\boldsymbol{\theta}, \boldsymbol{l}, \boldsymbol{u})$ where $f$ was defined in equation (1).

Function SCStime $\left(\theta_{1}, \theta_{2}, k_{1}, k_{2}, k_{3}\right)$

Step 1. Let $\boldsymbol{a}=\left(x_{a}, y_{a}\right):=D\left(\mathcal{C}_{k_{1}}\left(\theta_{s}, \theta_{1}\right)\right)+D\left(\mathcal{C}_{k_{2}}\left(\theta_{1}, \theta_{2}\right)\right)+D\left(\mathcal{C}_{k_{3}}\left(\theta_{2}, \theta_{t}\right)\right)$.

Step 2. Let:

$$
\begin{aligned}
& l_{1}:=\frac{\left(x_{t}-x_{a}\right) \sin \theta_{2}-\left(y_{t}-y_{a}\right) \cos \theta_{2}}{\sin \left(\theta_{2}-\theta_{1}\right)}, \\
& l_{2}:=\frac{\left(x_{t}-x_{a}\right) \sin \theta_{1}-\left(y_{t}-y_{a}\right) \cos \theta_{1}}{\sin \left(\theta_{1}-\theta_{2}\right)} .
\end{aligned}
$$

Step 3.• If $l_{1}<0$ or $l_{2}<0$, the path is unfeasible: return $\tau=+\infty$.

- Else return the path time $\tau:=f(\boldsymbol{\theta}, \boldsymbol{l}, \boldsymbol{u})$.

Table 1: Function SCStime $\left(\theta_{1}, \theta_{2}, k_{1}, k_{2}, k_{3}\right)$.

It was assumed that the speed polar plot has a finite number of bitangents, which using Proposition 2 implies that there are only finitely many candidate $\mathcal{S C S}$ paths. In practice, this means that the pairs $\left(\theta_{1}, \theta_{2}\right)$ of angles such that the line passing through $\left(\theta_{1}, V\left(\theta_{1}\right)\right)$ and $\left(\theta_{2}, V\left(\theta_{2}\right)\right)$ is a convex bitangent to the speed polar plot can be enumerated. As a result one may need to evaluate all candidate paths to identify the optimal $\mathcal{S C S}$ path. 


\subsection{Construction of $\mathcal{S C C}$ paths}

It was mentioned that the main issue in implementing an enumerative algorithm, as it is done in most similar problems, stems from the presence of the $\mathcal{S C C}$ paths. Since all the candidate $\mathcal{S C C}$ paths cannot be listed, an alternative way to find the candidate optimal paths must be looked for. Therefore it is shown that it is possible to carry out this search by performing several gradient descent algorithms. The problem of fitting a $\mathcal{S C C}$ path with a pre-specified straight-line heading $\theta_{1}$ is first considered. It is then shown that given a feasible $\mathcal{S C C}$ path with straight-line heading $\theta_{1}$, there exists a neighborhood of $\theta_{1}$ for which $\mathcal{S C C}$ paths with a straight-line heading in that neighborhood are feasible and in which a gradient descent algorithm can be performed to find a candidate optimal $\mathcal{S C C}$ path and the corresponding $\theta_{1}$. Finally, it is shown that there exists a finite number of such intervals and a way to find them is discussed. Throughout this section, the focus is on $\mathcal{S C C}$ paths of the form $\mathcal{C}_{k_{1}}\left(\theta_{s}, \theta_{1}\right) \mathcal{S C}_{k_{2}}\left(\theta_{1}, \theta_{2}\right) \mathcal{C}_{k_{3}}\left(\theta_{2}, \theta_{t}\right)$, as opposed to $\mathcal{C}_{k_{1}}\left(\theta_{s}, \theta_{1}\right) \mathcal{C}_{k_{2}}\left(\theta_{1}, \theta_{2}\right) \mathcal{S C}_{k_{3}}\left(\theta_{2}, \theta_{t}\right)$ for which a similar reasoning holds.

\subsubsection{Feasibility of $\mathcal{S C C}$ paths with fixed straight-line heading}

Instead of looking for an optimal $\mathcal{S C C}$ path, consider the case of fixing the straight-line segment's heading and answer the question of whether it is possible to fit a $\mathcal{S C C}$ path with such a straight-line heading. Suppose then that the straight line segment's heading is fixed to $\theta_{1}$ and the sharpest turns' directions to $k_{1}, k_{2}, k_{3}$, and one tries to fit, without loss of generality, a $\mathcal{S C C}$ path of the form $\mathcal{C}_{k_{1}}\left(\theta_{s}, \theta_{1}\right) \mathcal{S C}_{k_{2}}\left(\theta_{1}, \theta_{2}\right) \mathcal{C}_{k_{3}}\left(\theta_{2}, \theta_{t}\right)$. In order to fully characterize this path, when such path is feasible, the remaining parameters need to be specified, that is, determine the length of the straight line segment, as well as the switching angle $\theta_{2}$ between the two consecutive turns $\mathcal{C}_{k_{2}}\left(\theta_{1}, \theta_{2}\right)$ and $\mathcal{C}_{k_{3}}\left(\theta_{2}, \theta_{t}\right)$. This task is more complicated than fitting two straight lines in the $\mathcal{S C S}$ case. Let $\boldsymbol{a}=\left(x_{a}, y_{a}\right):=D\left(\mathcal{C}_{k_{1}}\left(\theta_{s}, \theta_{1}\right)\right)$ be the displacement achieved by the first turn $\mathcal{C}_{k_{1}}\left(\theta_{s}, \theta_{1}\right), \boldsymbol{v}_{\theta_{1}}:=\left(\cos \theta_{1}, \sin \theta_{1}\right)$ be the direction vector of the straight line segment and $g: \theta \mapsto\left(x_{t}, y_{t}\right)-\left[D\left(\mathcal{C}_{k_{2}}\left(\theta_{1}, \theta\right)\right)+D\left(\mathcal{C}_{k_{3}}\left(\theta, \theta_{t}\right)\right)\right]$. Using these notations, the problem faced is one of finding $l_{1} \geq 0$ and $\theta_{2}$ such that:

$$
\boldsymbol{a}+l_{1} \boldsymbol{v}_{\theta_{1}}=g\left(\theta_{2}\right) .
$$

Figure 8 illustrates this problem, where the dashed line represents part of the locus of points described by $g$. A solution $\theta_{2}$ to equation $(16$ is an angle $\theta$ for which the graph of $g$ intersects the half-line starting at $\boldsymbol{a}$ with direction $\boldsymbol{v}_{\theta_{1}}$. It should be noted that there could be several solutions to this problem and that when trying to find the candidate path with minimum travel time, all solutions should be evaluated in order to select the fastest one.

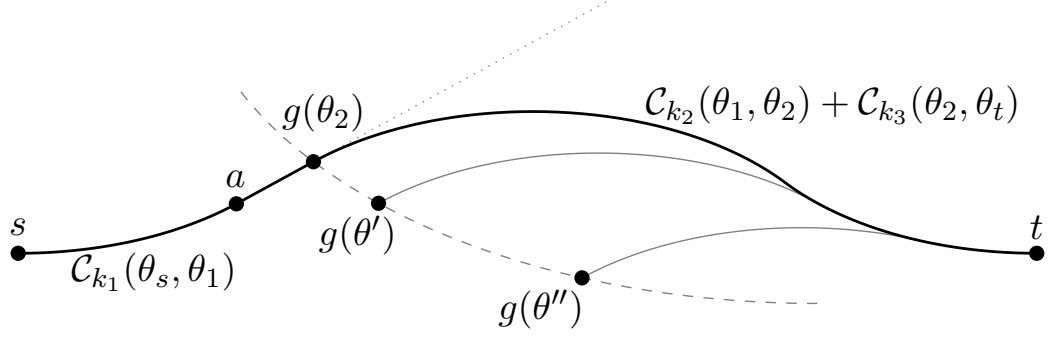

Figure 8: Construction of $\mathcal{S C C}$ paths

Table 2 presents the pseudo-code of a function $\operatorname{SCCtime}\left(\theta_{1}, k_{1}, k_{2}\right)$ that takes as inputs the heading $\theta_{1}$ of the straight line and the directions of the first and second turns $k_{1}$ and $k_{2}$, respectively, and outputs the travel time of the fastest corresponding $\mathcal{C S C C}$ path. A similar function $\operatorname{CCStime}\left(\theta_{1}, k_{1}, k_{3}\right)$ is defined to compute the travel time for the corresponding $\mathcal{C C S C}$ path. 
Function $\operatorname{SCCtime}\left(\theta_{1}, k_{1}, k_{2}\right)$

Step 1. Let $\boldsymbol{a}=\left(x_{a}, y_{a}\right):=D\left(\mathcal{C}_{k_{1}}\left(\theta_{s}, \theta_{1}\right)\right)$.

Step 2. Solve:

$$
\begin{array}{ll} 
& a+l_{1} \boldsymbol{v}_{\theta_{1}}=g\left(\theta_{2}\right) \\
\text { s.t. } & l_{1}>0
\end{array}
$$

and let $\mathcal{B}$ be the set of $\left(\theta_{2}, l_{1}\right)$ solutions to this problem.

Step 3.• If $\mathcal{B}=\emptyset$, no feasible $\mathcal{C S C C}$ path with a straight-line heading of $\theta_{1}$ exists: return $\tau=+\infty$.

- Else return the path time $\min _{\left(\theta_{2}, l_{1}\right) \in \mathcal{B}} f\left(\theta_{1}, \theta_{2}, l_{1}, 0, \boldsymbol{u}\right)$.

Table 2: Function $\operatorname{SCCtime}\left(\theta_{1}, k_{1}, k_{2}\right)$.

\subsubsection{Local feasibility of $\mathcal{S C C}$ paths}

Section 3.3.1 tackled the question of the feasibility of a $\mathcal{S C C}$ path of the form $\mathcal{C}_{k_{1}}\left(\theta_{s}, \theta_{1}\right) \mathcal{S C}_{k_{2}}\left(\theta_{1}, \theta_{2}\right) \mathcal{C}_{k_{3}}\left(\theta_{2}, \theta_{t}\right)$ for a given angle $\theta_{1}$. The next step to evaluate is whether, given an angle $\theta_{1}$ for which such a feasible path exists, one may deviate the straight-line segment's heading from $\theta_{1}$ while maintaining feasibility. This could be very helpful if true, for it would mean that if a feasible $\mathcal{S C C}$ path could be found with a straight-line heading $\theta_{1}$ close to an optimal $\mathcal{S C C}$ path with straight-line heading $\theta_{1}^{*}$, one might be able by performing some optimization method to converge to this optimal value.

However, if one wants to modify the straight-line segment's heading while preserving feasibility of the path, one must also alter the straight-line segment's length $l_{1}$ and the switching angle between the two consecutive turns $\theta_{2}$. Ideally, one would like to express these values as a function of $\theta_{1}$ so the problem can be treated, at least locally, as a minimization problem with respect to $\theta_{1}$ alone. This requires using the Implicit Function Theorem (see for instance Rudin 1976] [ch.9]). In order to apply it, equation (16) is rewritten as:

$$
\left\{\begin{array}{c}
D^{x}\left(\mathcal{C}_{k_{1}}\left(\theta_{s}, \theta_{1}\right)\right)+l_{1} \cos \theta_{1}+D^{x}\left(\mathcal{C}_{k_{2}}\left(\theta_{1}, \theta_{2}\right)\right)+D^{x}\left(\mathcal{C}_{k_{3}}\left(\theta_{2}, \theta_{t}\right)\right)=x_{t} \\
D^{y}\left(\mathcal{C}_{k_{1}}\left(\theta_{s}, \theta_{1}\right)\right)+l_{1} \sin \theta_{1}+D^{y}\left(\mathcal{C}_{k_{2}}\left(\theta_{1}, \theta_{2}\right)\right)+D^{y}\left(\mathcal{C}_{k_{3}}\left(\theta_{2}, \theta_{t}\right)\right)=y_{t}
\end{array}\right.
$$

or equivalently, letting $\boldsymbol{q}:=\left(\theta_{2}, l_{1}\right)$ :

$$
h\left(\theta_{1}, \boldsymbol{q}\right)=\left(x_{t}, y_{t}\right)
$$

where $h$ is defined as:

$$
h\left(\theta_{1}, \boldsymbol{q}\right):=\left(\begin{array}{l}
D^{x}\left(\mathcal{C}_{k_{1}}\left(\theta_{s}, \theta_{1}\right)\right)+l_{1} \cos \theta_{1}+D^{x}\left(\mathcal{C}_{k_{2}}\left(\theta_{1}, \theta_{2}\right)\right)+D^{x}\left(\mathcal{C}_{k_{3}}\left(\theta_{2}, \theta_{t}\right)\right) \\
D^{y}\left(\mathcal{C}_{k_{1}}\left(\theta_{s}, \theta_{1}\right)\right)+l_{1} \sin \theta_{1}+D^{y}\left(\mathcal{C}_{k_{2}}\left(\theta_{1}, \theta_{2}\right)\right)+D^{y}\left(\mathcal{C}_{k_{3}}\left(\theta_{2}, \theta_{t}\right)\right)
\end{array}\right) .
$$

The Jacobians of $h$ with respect to $\boldsymbol{q}$ and $\theta_{1}$ read:

$$
\begin{aligned}
& \nabla_{\boldsymbol{q}} h\left(\theta_{1}, \boldsymbol{q}\right)=\left(\begin{array}{ll}
\left(u_{2}-u_{3}\right) R\left(\theta_{2}\right) \cos \theta_{2} & \cos \theta_{1} \\
\left(u_{2}-u_{3}\right) R\left(\theta_{2}\right) \sin \theta_{2} & \sin \theta_{1}
\end{array}\right)=\left(\begin{array}{ll}
2 u_{2} R\left(\theta_{2}\right) \cos \theta_{2} & \cos \theta_{1} \\
2 u_{2} R\left(\theta_{2}\right) \sin \theta_{2} & \sin \theta_{1}
\end{array}\right) \\
& \nabla_{\theta_{1}} h\left(\theta_{1}, \boldsymbol{q}\right)=\left(\begin{array}{l}
\left(u_{1}-u_{2}\right) R\left(\theta_{1}\right) \cos \theta_{1}-l_{1} \sin \theta_{1} \\
\left(u_{1}-u_{2}\right) R\left(\theta_{1}\right) \sin \theta_{1}+l_{1} \cos \theta_{1}
\end{array}\right)
\end{aligned}
$$

where $u_{i}=-1$ (respectively +1 ) when $k_{i}=L$ (respectively $R$ ) for $i=1,2,3$.

Consider a feasible $\mathcal{S C C}$ path characterized by turns, angles and straight-line segment length $u_{1}, u_{2}, u_{3}, \theta_{1}, \theta_{2}, l_{1}$. This implies in particular that $\theta_{1} \neq \theta_{2}$ and $l_{1}>0$, which, in turn, implies that the Jacobian of $h$ with respect to $\boldsymbol{q}$ is invertible. The invertibility of $\nabla_{\boldsymbol{q}} h$ at $\left(\theta_{1}, \boldsymbol{q}\right)$ justifies the application of the Implicit Function Theorem and state that there exists a neighborhood $\mathcal{V}$ of $\theta_{1}$ such that there exists a differentiable function 
$\varphi: \theta \mapsto\left(\varphi_{1}(\theta), \varphi_{2}(\theta)\right)$ defined on $\mathcal{V}$ such that for any $\theta \in \mathcal{V}$ :

$$
\begin{aligned}
h(\theta, \varphi(\theta)) & =\left(x_{t}, y_{t}\right), \\
\nabla \varphi(\theta) & =\left(\begin{array}{l}
\varphi_{1}^{\prime}\left(\theta_{1}\right) \\
\varphi_{2}^{\prime}\left(\theta_{1}\right)
\end{array}\right)=-\left(\nabla_{\boldsymbol{q}} h(\theta, \boldsymbol{q})\right)^{-1} \nabla_{\theta_{1}} h(\theta, \boldsymbol{q}) .
\end{aligned}
$$

It is also required that the length of the straight-line segment be positive, and thus, that $\varphi_{2}(\theta)>0$. Since $\varphi_{2}\left(\theta_{1}\right)=l_{1}>0$, the continuity of $\varphi$ guarantees that there exists a neighborhood $\mathcal{W} \subseteq \mathcal{V}$ on which (17) and (18) are satisfied, and further $\varphi_{2}(\theta)>0$.

The meaning of these properties is that, given a feasible $\mathcal{S C C}$ path, it is possible to locally continuously deform it while maintaining a feasible $\mathcal{S C C}$ structure. Furthermore, all changes in the structure of the resulting optimal path can be expressed as functions of the straight-line's heading alone.

\subsubsection{Local optimization of $\mathcal{S C C}$ paths}

In Section 3.3.2, it was shown that given a feasible $\mathcal{S C C}$ path with straight-line segment's heading $\theta_{1}$, one could freely tweak the straight-line segment's heading within a neighborhood of $\theta_{1}$ while maintaining $\mathcal{S C C}$ feasibility. In this section the focus is on the problem of finding an optimal $\mathcal{S C C}$ within such neighborhood. It is thus assumed in this section that a feasible $\mathcal{S C C}$ path is given, from which an optimization scheme can be started.

Consider a feasible $\mathcal{S C C}$ path characterized by turn directions $u_{1}, u_{2}, u_{3}$, a straight-line segment heading $\theta_{1}^{0}$, straight-line segment length $l_{1}^{0}$ and a switching heading between the two consecutive turns $\theta_{2}^{0}$. The travel time corresponding to this path is given by $f\left(\boldsymbol{\theta}^{0}, \boldsymbol{l}^{0}, \boldsymbol{u}\right)$, where $l_{2}^{0}=0$.It was shown that there exists an open interval $\mathcal{W}$ containing $\theta_{1}^{0}$ such that for any $\theta \in \mathcal{W}$ :

$$
\begin{aligned}
h(\theta, \varphi(\theta)) & =\left(x_{t}, y_{t}\right), \\
\varphi_{2}(\theta) & >0 .
\end{aligned}
$$

Our goal is to find $\theta_{1}^{*} \in \mathcal{W}$ such that $\theta_{1}^{*}$ is a local minimizer of $\tilde{f}$, if such $\theta_{1}^{*}$ exists:

$$
\tilde{f}\left(\theta_{1}^{*}\right)=\min _{\theta_{1} \in \mathcal{W}} \tilde{f}\left(\theta_{1}\right)
$$

where $\tilde{f}$ is a function of $\theta_{1}$ defined as:

$$
\tilde{f}\left(\theta_{1}\right)=f\left(\theta_{1}, \varphi_{1}\left(\theta_{1}\right), \varphi_{2}\left(\theta_{1}\right), 0, \boldsymbol{u}\right)
$$

To solve this problem, a gradient descent algorithm is used. It converges to a minimum if one exists, or to a boundary point of $\mathcal{W}$. These problems of existence and uniqueness of minima are solved in the next section and the focus is here on deriving the tools necessary to the implementation of the gradient descent.

The basic step of the gradient descent algorithm updates the current estimate $\theta_{1}^{n}$ of $\theta_{1}^{*}$ as:

$$
\theta_{1}^{n+1}=\theta_{1}^{n}-\eta_{n} \frac{d \tilde{f}}{d \theta_{1}}\left(\theta_{1}^{n}\right)
$$

where $\eta_{n}$ is the step size.

In order to implement the gradient descent algorithm, the derivative $\frac{d \tilde{f}}{d \theta_{1}}$ needs to be computed. Recall that $f$ was defined in (1) and thus:

$$
\tilde{f}\left(\theta_{1}\right)=T\left(\theta_{s}, \theta_{1}, u_{1}\right)+\frac{\varphi_{2}\left(\theta_{1}\right)}{V\left(\theta_{1}\right)}+T\left(\theta_{1}, \varphi_{1}\left(\theta_{1}\right), u_{2}\right)+T\left(\varphi_{1}\left(\theta_{1}\right), \theta_{t}, u_{3}\right) .
$$

Differentiating $\tilde{f}$ with respect to $\theta_{1}$ then yields:

$$
\frac{d \tilde{f}}{d \theta_{1}}\left(\theta_{1}\right)=\left(u_{1}-u_{2}\right) \frac{R\left(\theta_{1}\right)}{V\left(\theta_{1}\right)}+\frac{\varphi_{2}^{\prime} V\left(\theta_{1}\right)-V^{\prime}\left(\theta_{1}\right) \varphi_{2}\left(\theta_{1}\right)}{V\left(\theta_{1}\right)^{2}}+\left(u_{2}-u_{3}\right) \varphi_{1}^{\prime}\left(\theta_{1}\right) \frac{R\left(\theta_{2}\right)}{V\left(\theta_{2}\right)},
$$

where $\varphi_{1}^{\prime}\left(\theta_{1}\right)$ and $\varphi_{2}^{\prime}\left(\theta_{1}\right)$ are obtained by computing (18).

Our implementation of the gradient descent algorithm for $\mathcal{S C C}$ paths with turn directions $u_{1}, u_{2}, u_{3}$ thus works as follows: 
1. Start with a feasible $\mathcal{S C C}$ path characterized by a straight-line segment with heading $\theta_{1}^{0}$ and length $l_{1}^{0}$ and switching angle between the two consecutive turns $\theta_{2}^{0}$. Set $n=0$.

2. At the $n+1$-th iteration, choose a step-size $\eta_{n}$ and tentatively update the current estimate of $\theta_{1}^{*}$ :

$$
\hat{\theta}_{1}^{n+1}=\theta_{1}^{n}-\eta_{n} \frac{d \tilde{f}}{d \theta_{1}}\left(\theta_{1}^{n}\right) .
$$

3. Solve 160 for $\theta_{2}^{n+1}$ and $l_{1}^{n+1}$ :

$$
\begin{array}{ll} 
& a+l_{1}^{n+1} \boldsymbol{v}_{\hat{\theta}_{1}}=g\left(\theta_{2}^{n+1}\right) \\
\text { s.t. } & l_{1}^{n+1}>0
\end{array}
$$

If the problem does not have a solution, decrease $\eta_{n}$ and go back to step 2 with $\theta_{1}^{n}$ as the current estimate of $\theta_{1}^{*}$. Else accept $\theta_{1}^{n+1}=\hat{\theta}_{1}^{n+1}$, set $n=n+1$ and go back to step 2 .

4. Stop when either $\left|\frac{d \tilde{f}}{d \theta_{1}}\left(\theta_{1}^{n+1}\right)\right|<\varepsilon_{1}$, or $\left|\theta_{1}^{n+1}-\theta_{1}^{n}\right|<\varepsilon_{2}$ or $n>N$, for given stopping criteria $\varepsilon_{1}>0$, $\varepsilon_{2}>0$ and $N>0$.

Implementation example To illustrate the procedure, it is applied to a particular example. Consider a speed polar plot derived from the piecewise linear speed polar plot presented in Figure 1, which is adapted from Dolinskaya et al. 2009. The speed corresponds to the S175 containership in sea state 7, and the corresponding minimum-turning radius function is used, which is a function of the vessel's speed. Instead of linearly interpolating the data points, a cubic spline interpolation is chosen, so as to fit in the scope of the current problem and obtain a differentiable speed function. The resulting speed polar plot is displayed in Figure 9

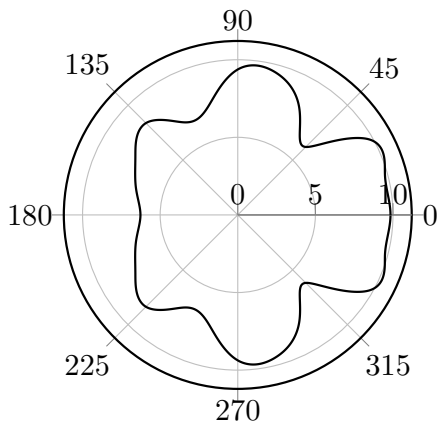

Figure 9: Differentiable speed polar plot obtained by cubic spline interpolation of the data points from the S175 containership in Sea State 7 (adapted from Dolinskaya et al. 2009]).

Consider initial and final configurations given by:

$$
\left(x_{s}, y_{s}, \theta_{s}\right)=\left(0,0,315^{\circ}\right) \quad \text { and } \quad\left(x_{t}, y_{t}, \theta_{t}\right)=\left(L \cos \left(315^{\circ}\right), L \sin \left(315^{\circ}\right), 315^{\circ}\right),
$$

for $L=500$ meters. In other words a distance of 500 meters along a line with a direction $315^{\circ}$ is traveled.

Consider $\mathcal{S C C}$ paths of the type $\mathcal{C}_{L}\left(\theta_{s}, \theta_{1}\right) \mathcal{S C}_{R}\left(\theta_{1}, \theta_{2}\right) \mathcal{C}_{L}\left(\theta_{2}, \theta_{t}\right)$ and start with a feasible path having a straight-line segment heading of $\theta_{1}^{0}=316^{\circ}$. The gradient descent algorithm converges to a candidate optimal path having a straight-line segment heading $\theta_{1}^{*}$. Figure 10 shows on the top graph (with rotated axes so that the $x$-axis has a direction of $315^{\circ}$ ) the evolution of the path through the algorithm's iterations from the initial feasible path to the candidate optimal path, with selected intermediate paths in between. The bottom graph shows the evolution of the points $\left(\theta_{1}^{n}, V\left(\theta_{1}^{n}\right)\right)$ and $\left(\theta_{2}^{n}, V\left(\theta_{2}^{n}\right)\right)$ on the speed polar plot as the algorithm progresses. It is particularly interesting to observe how the line joining these points shifts to eventually be tangent to the speed polar plot at $\left(\theta_{1}^{*}, V\left(\theta_{1}^{*}\right)\right)$, as required by Proposition 3 . 

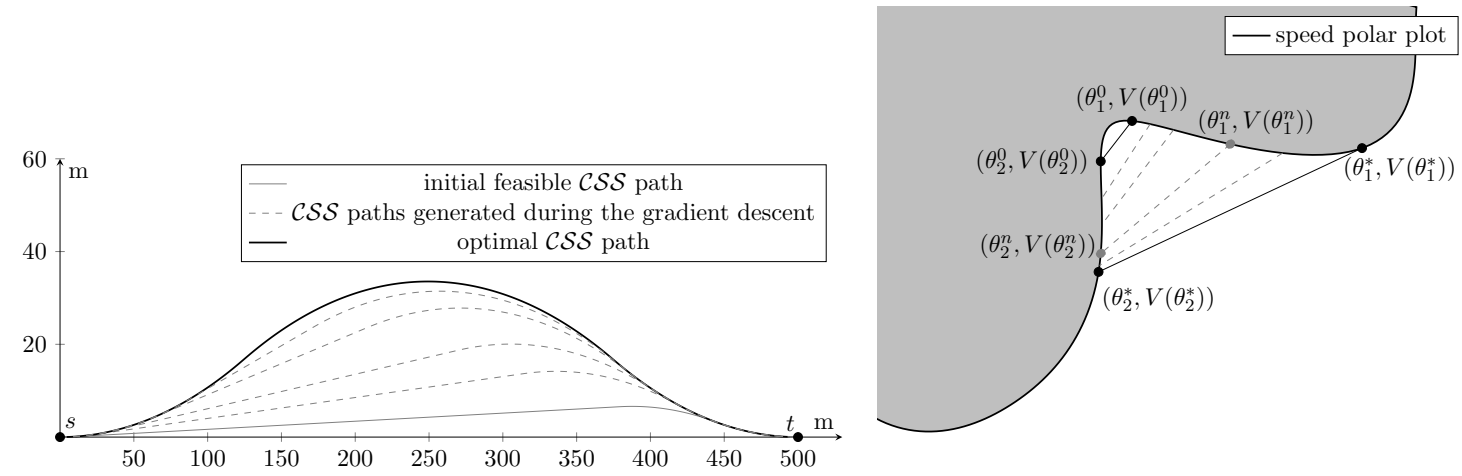

Figure 10: Illustrations of the gradient descent example: (left) evolution of the candidate path, (right) evolution of the points $\left(\theta_{1}^{n}, V\left(\theta_{1}^{n}\right)\right)$ and $\left(\theta_{2}^{n}, V\left(\theta_{2}^{n}\right)\right)$ on the speed polar plot.

\subsubsection{Global optimization of $\mathcal{S C C}$ paths}

It was shown in Sections 3.3 .2 and 3.3 .3 that if a feasible $\mathcal{S C C}$ path with straight-line heading $\theta_{1}$ was found, the path could be continuously deformed such that it remained a $\mathcal{S C C}$ path with a straight-line heading belonging to a neighborhood $\mathcal{W}$ of $\theta_{1}$. Furthermore, a method that either finds a local minimum in $\mathcal{W}$ or converges to its boundary was derived.

The problem now becomes one of finding the intervals of $S^{1}$ such that for any $\theta_{1}$ in one such interval there exist feasible $\mathcal{S C C}$ paths with a straight-line heading $\theta_{1}$. Consider an open interval $\mathcal{W}$ as described above. For any $\theta_{1} \in \mathcal{W}, h\left(\theta_{1}, \varphi\left(\theta_{1}\right)\right)=\left(x_{t}, y_{t}\right)$. Then, consider a sequence $\left\{\theta_{1}^{i}\right\}_{i} \in \mathcal{W}^{\mathbb{N}}$ converging to a boundary point $\theta_{1}^{b}$ of $\mathcal{W}$ and let $\theta_{2}^{b}:=\lim _{i} \varphi_{1}\left(\theta_{1}^{i}\right)$ and $l_{1}^{b}:=\lim _{i} \varphi_{2}\left(\theta_{1}^{i}\right)$. By continuity of $h$ and $\varphi$, we must also have $h\left(\theta_{1}^{b}, \theta_{2}^{b}, l_{1}^{b}\right)=\left(x_{t}, y_{t}\right)$. However, $\theta_{1}^{b}$ being on the boundary of $\mathcal{W}$, the path characterized by $\theta_{1}^{b}, \theta_{2}^{b}$ and $l_{1}^{b}$ cannot be a feasible $\mathcal{S C C}$ path. It thus corresponds to a path where the continuous deformation of a $\mathcal{S C C}$ path either degenerates or reaches a constraint limit. This can happen if one of the following holds: $\theta_{1}^{b}=\theta_{s}^{b}, \theta_{1}^{b}=\theta_{2}^{b}, \theta_{2}^{b}=\theta_{t}^{b}$ or $l_{1}=0$. Note that the limiting cases corresponding to the angular equalities above can be reached in two different ways, either when a sharpest turn vanishes, or when a turn is a full $2 \pi$-turn.

Conversely, by using similar arguments to the one used in Section 3.3.2 if one of these limiting cases exists, it can be continuously transformed into a $\mathcal{S C C}$ path. This yields a way of finding the intervals in which one can find $\theta_{1}$ such that a feasible $\mathcal{S C C}$ path with heading $\theta_{1}$ can be constructed.

Let $\mathcal{F}$ be the collection of such intervals. The remaining question is that of the number of local optima in any interval $\mathcal{W} \in \mathcal{F}$. To tackle this question, recall that according to Proposition 4 the straight-line heading of an optimal $\mathcal{S C C}$ path is a non-bordering convex angle. Letting $\mathcal{N}$ be the collection of non-bordering convex intervals, it is already known that one can restrict their search of an optimal $\mathcal{S C C}$ path to the intervals in $\mathcal{F} \cap \mathcal{N}$. It can further be stated that any element of $\mathcal{F} \cap \mathcal{N}$ contains at most one local optimum. This can be seen from the fact that a necessary condition for a $\mathcal{S C C}$ path to be a local maximum is that its straight-line heading be a non-bordering concave angle. Consequently, if there were more than one local optimum in an element of $\mathcal{F} \cap \mathcal{N}$, then there should exist at least one local maximum, which would contradict the fact that the elements of $\mathcal{F} \cap \mathcal{N}$ are non-bordering convex intervals.

The global optimal $\mathcal{S C C}$ path can thus be found by applying the gradient descent method developed in Section 3.3 .3 in all the elements of $\mathcal{F} \cap \mathcal{N}$.

Example The above procedure is illustrated using the same speed polar plot and minimum-turning radius as in the example developed in Section 3.3.3 (see Figure 9). An initial heading $\theta_{s}=0$ and a final configuration $\left(x_{t}, y_{t}, \theta_{t}\right)=(400,800,2 \pi / 3)$ are used. For this problem, it is found that the interval $I=(0.07,1.88)$ belongs to $\mathcal{F}$, where the limiting cases $\theta_{1}=0.07$ corresponds to the first sharpest turn being a full $2 \pi$-turn $\left(\theta_{1}=\theta_{s}\right)$ and $\theta_{1}=1.88$ corresponds to a degenerate case where the final turn vanishes $\left(\theta_{2}=\theta_{t}\right)$. Intersecting $I$ with the elements of $\mathcal{N}$ yields three intervals $I_{1}=(0.07,0.139], I_{2}=[0.299,0.702]$ and $I_{3}=[0.945,1.793)$. The gradient descent scheme thus needs to be applied on all three intervals. 
1. Starting the gradient descent with $\theta_{1}^{0}=0.139$ produces an optimal path on $I_{1}=(0.07,0.139]$ characterized by $\left(\theta_{1}^{*}, \theta_{2}^{*}, l_{1}^{*}\right)=(0.107,0.202,189.75)$ and a travel time of $513.22 \mathrm{~s}$.

2. Starting the gradient descent with $\theta_{1}^{0}=0.702$ produces an optimal path on $I_{2}=[0.299,0.702]$ characterized by $\left(\theta_{1}^{*}, \theta_{2}^{*}, l_{1}^{*}\right)=(0.567,0.899,400.67)$ and a travel time of $501.07 \mathrm{~s}$.

3. Starting the gradient descent with $\theta_{1}^{0}=0.945$ produces an optimal path on $I_{3}=[0.945,1.793)$ characterized by $\left(\theta_{1}^{*}, \theta_{2}^{*}, l_{1}^{*}\right)=(1.633,2.1067,430.05)$ and a travel time of $489.57 \mathrm{~s}$.

Figure 11 shows the evolution of $\theta_{1}^{n}$ and $\theta_{2}^{n}$ during the gradient descent in $I_{2}$ and $I_{3}$. Observe that the local optima are indeed reached for straight line headings $\theta_{1}$ such that the tangent to the speed polar plot at $\theta_{1}$ coincides with the line joining $\left(\theta_{1}, V\left(\theta_{1}\right)\right)$ and $\left(\theta_{2}, V\left(\theta_{2}\right)\right)$ (in polar coordinates).
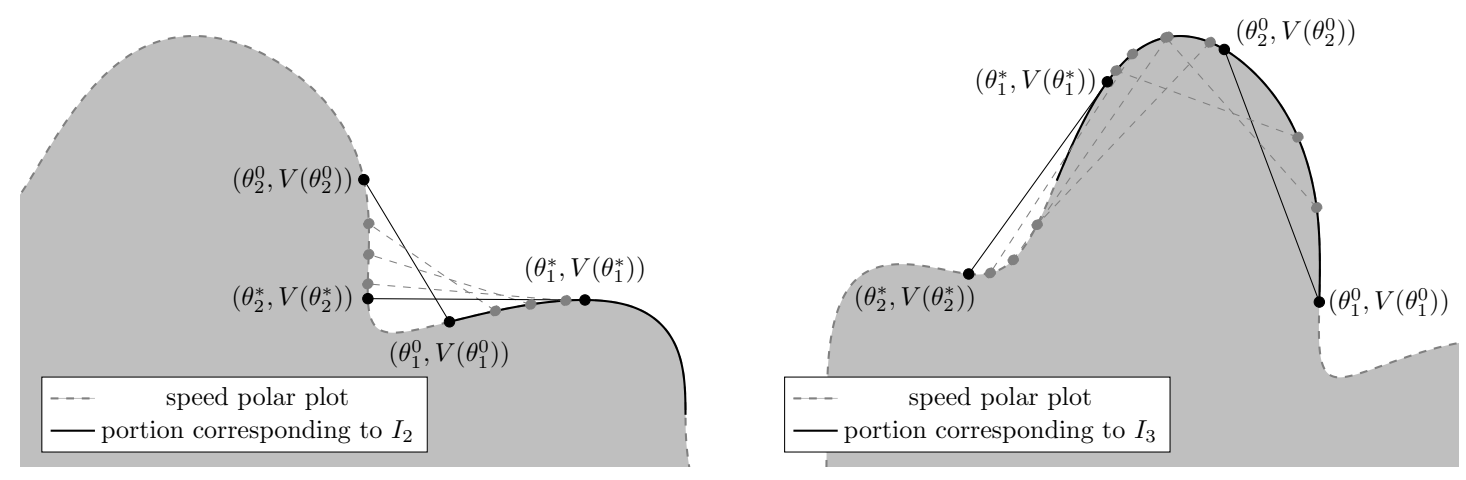

Figure 11: Evolution of $\theta_{1}^{n}$ and $\theta_{2}^{n}$ during the gradient descent in $I_{2}$ and $I_{3}$

\subsection{Formulation of the fastest-path finding algorithm for differentiable speed functions}

In this section, the results obtained in Sections $3.1,3.2$ and 3.3 are summarized, facilitating the solution to the original problem of finding a fastest path between two configurations $\left(x_{s}, y_{s}, \theta_{s}\right)$ and $\left(x_{t}, y_{t}, \theta_{t}\right)$.

The development of the results has been based on the partitioning of the candidate optimal paths into three groups: the Dubins-like, $\mathcal{S C S}$ and $\mathcal{S C C}$ paths. The reasoning has then been to consider each category independently and work towards an optimal path.

Dubins-like paths: Section 3.1 recalled the algorithm from Dolinskaya and Maggiar 2012 that solves for the optimal Dubins-like path.

$\mathcal{S C S}$ paths: It was shown in Section 3.2 how, given the three turns' directions $\left(k_{1}, k_{2}\right.$ and $\left.k_{3}\right)$ and the two straight-line segments' headings $\theta_{1}$ and $\theta_{2}$, the travel time of a candidate $\mathcal{S C S}$ path could be computed; this resulted in the function SCStime. It was also shown in Section 2.2 that a necessary condition for a $\mathcal{S C S}$ path to be optimal is that the line passing through $\left(\theta_{1}, V\left(\theta_{1}\right)\right)$ and $\left(\theta_{2}, V\left(\theta_{2}\right)\right)$ (in polar coordinates) be a convex bitangent to the speed polar plot (cf. Proposition 2 ).

Provided the speed polar plot only has finitely many convex bitangents, it follows that it is possible to enumerate all the candidate $\mathcal{S C S}$ paths and evaluate them using the function SCStime. This implies looping through all the possible combinations of $k_{1}, k_{2}$ and $k_{3}$.

$\mathcal{S C C}$ paths: The $\mathcal{S C C}$ paths proved to be more troublesome for there is no explicit way to enumerate the candidate paths of this type, a departure from Dubins-like and $\mathcal{S C S}$ paths where this was the method of choice. It was however shown in Section 3.3 that given two turns' directions $k$ and $k^{\prime}$ (the choice of the other's turn direction is fixed for a $\mathcal{S C C}$ path), one could apply a finite number of optimization procedures to find the optimal $\mathcal{S C C}$ paths having the specified turn directions. Recall that there are two types of $\mathcal{S C C}$ paths, depending on whether the straight line segment is located after the first or second turn $(\mathcal{C S C C}$ versus $\mathcal{C C S C})$, and that the procedure must be applied to both types. 
These steps are put together in Algorithm 1, which takes as inputs: a speed and minimum-turning radius function, and an initial and a final configuration; and outputs the fastest path and the corresponding travel time.

Algorithm 1 Fastest Path with Bounded Curvature for a Differentiable Speed Function.

Step 0 Set $\tau, \tau_{1}, \tau_{2}, \tau_{3}$ to $\infty$.

Step 1 Apply Algorithm 1 from Dolinskaya and Maggiar 2012 that solves for Dubins-like paths and let $\tau_{1}$ be its output.

Let $\tau=\min \left(\tau, \tau_{1}\right)$.

\section{Step 2}

Step 2a Compute the set $\mathcal{D}$ of pairs of angles $\left(\theta_{1}, \theta_{2}\right)$ such that the line passing through $\left(\theta_{1}, V\left(\theta_{1}\right)\right)$ and $\left(\theta_{2}, V\left(\theta_{2}\right)\right)$ (in polar coordinates) is a convex bitangent to the speed polar plot. (Note that this can be done offline, independently of starting and final configurations).

Step $2 \mathbf{b}$ For all $k_{1}, k_{2}, k_{3} \in\{L, R\}$ and for all $\left(\theta_{1}, \theta_{2}\right) \in \mathcal{D}$, let $\tau_{2}^{\prime}=\operatorname{SCStime}\left(\theta_{1}, \theta_{2}, k_{1}, k_{2}, k_{3}\right)$ and $\tau_{2}=\min \left(\tau_{2}, \tau_{2}^{\prime}\right)$.

Step 2c Let $\tau=\min \left(\tau, \tau_{2}\right)$.

Step 3

Step 3a For all $k, k^{\prime} \in\{L, R\}$, let $\tau_{3}^{\prime}$ and $\tau_{3}^{\prime \prime}$ be the minimum travel times of paths of the form $\mathcal{C}_{k} \mathcal{S C}_{k^{\prime}} \mathcal{C}_{\bar{k}^{\prime}}$ and $\mathcal{C}_{k} \mathcal{C}_{\bar{k}} \mathcal{S C}_{k^{\prime}}$ obtained by applying the procedure described in Section 3.3 , and let $\tau_{3}=\min \left(\tau_{3}, \tau_{3}^{\prime}, \tau_{3}^{\prime \prime}\right)$.

Step 3b Let $\tau=\min \left(\tau, \tau_{3}\right)$.

Step 4 Return $\tau$ and the corresponding optimal path.

\subsection{Algorithm improvements and implementation}

In Step 2 of Algorithm 1, all possible combinations of pairs of angles in the set $\mathcal{D}$ are looped over to evaluate the candidate $\mathcal{S C S}$ paths. It is in fact not necessary to consider all paths because some candidate paths can be ruled out using Proposition 20 from Dolinskaya and Maggiar 2012. As a result, one can add a function in the algorithm that applies those tests before executing the SCStime function. Such implementation can increase the speed of the step corresponding to the search for the optimal $\mathcal{S C S}$ path.

In addition, the algorithm and its sub-routines repetitively require the evaluation of integrals of the type

$\int_{\theta_{a}}^{\theta_{b}} V(\theta) \cos \theta d \theta, \int_{\theta_{a}}^{\theta_{b}} V(\theta) \sin \theta d \theta$ and $\int_{\theta_{a}}^{\theta_{b}} \frac{R(\theta)}{V(\theta)} d \theta$ in order to evaluate displacement and travel time, which can be computationally costly. To reduce the computational cost, one can pre-compute the tables containing these values by discretizing sharpest turns with a sufficiently small angle step $\Delta \theta$. This method replaces all integral evaluations with simple lookup tables and considerably reduces the run time of the algorithm.

Finally, it should be recalled from 2.1.1 that the heading $\theta$ refers to the medium-relative orientation of the vehicle, as opposed to its inertial orientation.

\section{Applications and numerical results}

\subsection{Differentiable speed function}

In this section results obtained using Algorithm 1 are presented. As basis for the examples, the same speed and minimum-turning radius functions as those already used in the illustrations in Sections 3.3 .3 and 3.3 .4 are used, which correspond to the speed polar plot displayed in Figure 9.

Given an initial and a final configurations, Algorithm 1 is applied to obtain the fastest path joining them. Note that in order to apply the algorithm and in particular function SCStime, one needs to obtain 
the bitangents to the speed polar plot, which are shown in Figure 12.

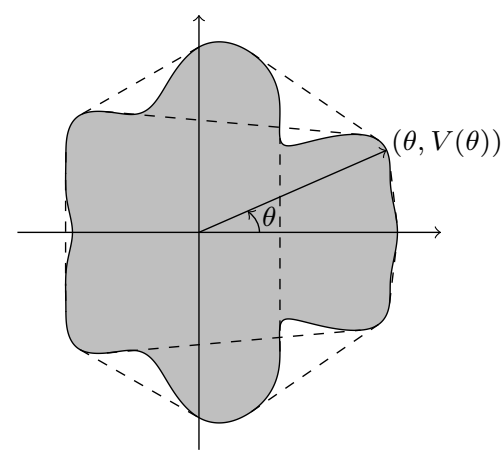

Figure 12: Bitangents of the differentiable speed polar plot used for the examples.

Figure 13 shows the fastest paths obtained for the following two sets of configurations: 1) $\theta_{s}=\pi / 3$ and $\left(x_{t}, y_{t}, \theta_{t}\right)=(-1500,500, \pi / 2)$, and 2$) \theta_{s}=2 \pi / 3$ and $\left(x_{t}, y_{t}, \theta_{t}\right)=(800,-1400, \pi / 4)$.

Two different behaviors are observed: while the second configuration yields an optimal path that corresponds to the Dubins-like solution, the optimal path for the first configuration is very different from the Dubins-like solution. For the first configuration, the optimal path path has a travel time of 500.3 seconds against 505.3 seconds for the Dubins-like path. Thus, restricting to Dubins-like paths could prove very sub-optimal, which underlines the relevance of this work.
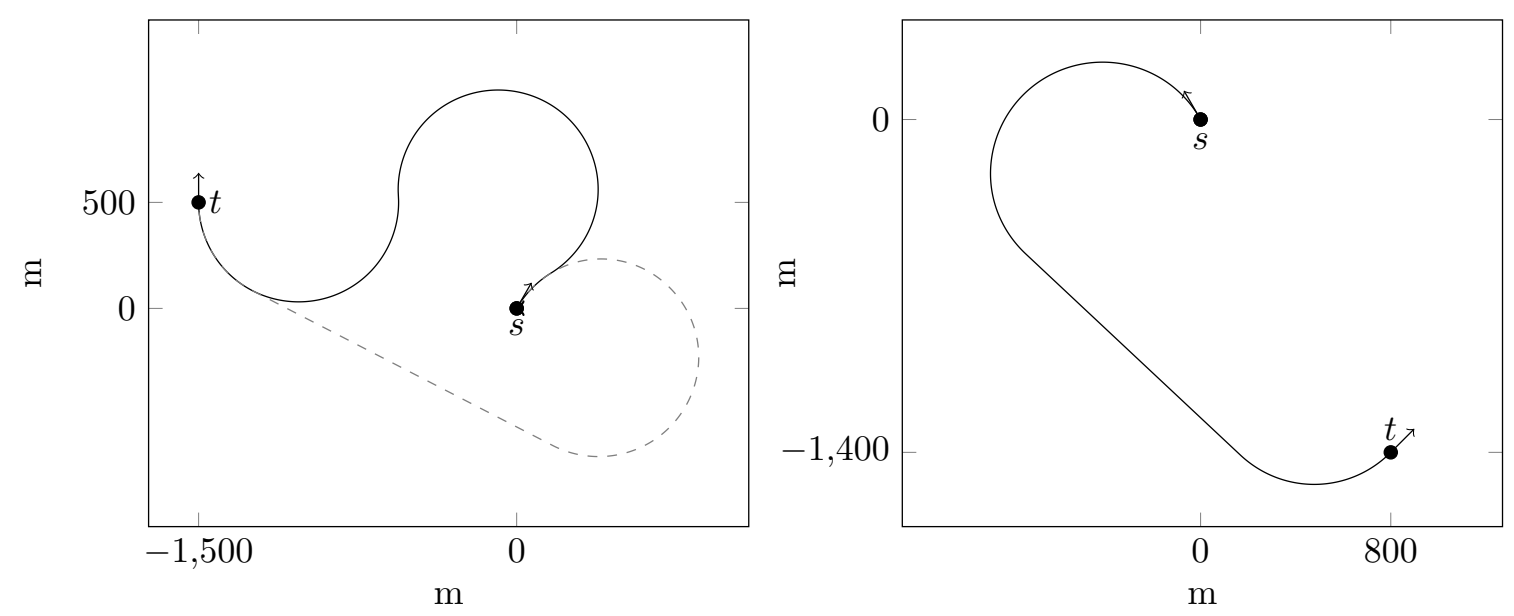

Figure 13: Examples of fastest-paths for the differentiable speed function compared to the Dubins-like fastest-paths (in dashed).

\subsection{Piecewise linear speed polar plot}

Piecewise linear speed polar plots are models of interest in their own right for they appear in concrete and real applications. For example, one of the motivating examples behind the work in Dolinskaya and Maggiar 2012 was the non-convex speed polar plot that arises when studying optimal short-range routing of a vessel in a stationary random seaway characterized by a sea state. The modeled speed polar plot that takes into account the physical constraints operating on a boat, and which are reproduced in Figure 1 in the case of the S175 containership, turns out to be non-convex and piecewise linear. As in many applications, the speed polar plot is constructed from discrete data that is then interpolated, yielding the observed piecewise linear speed polar plot. 
Additionally, any differentiable speed polar plot can be seen as the limit of a sequence of piecewise linear speed polar plots. Limiting arguments to approximate fastest paths of vessels with differentiable speed functions by piecewise linear speed polar plots can be used. Hence, solving the problem for piecewise linear speed polar plots solves the more general problem with arbitrary precision. This can help ease the implementation of the algorithm derived in Section 3.

In the case of piecewise linear speed polar plots, it can be easily shown that the straight line segment's headings, be it for a $\mathcal{S C S}$ or $\mathcal{S C C}$ path, correspond to break-points of the speed polar plot. This observation greatly facilitates the implementation for it waives the necessity of the optimization procedure derived in Section 3.3 .

The nonconvex speed polar plot of the S175 containership in sea state 7 displayed in Figure 1 has been one of the main motivating problems behind the work presented here. In this section the results obtained for that piecewise linear speed polar plot are presented. In Figure 14 two examples of fastest-paths are plotted and compared to what would have been obtained restricting to Dubins-like paths. They correspond to the following configurations: 1) $\theta_{s}=\pi / 4$ and $\left(x_{t}, y_{t}, \theta_{t}\right)=(-1500,-500, \pi / 4)$ (optimal path travel time: $596.5 \mathrm{~s}$, Dubins-like travel time: $597.2 \mathrm{~s})$; and 2) $\theta_{s}=\pi / 4$ and $\left(x_{t}, y_{t}, \theta_{t}\right)=(-1500,-500, \pi / 2)$ (optimal path travel time: $530.9 \mathrm{~s}$, Dubins-like travel time: $536.9 \mathrm{~s})$.

It can be observed that the fastest path in the case of a non-convex speed polar plot can be in some cases very different to the fastest path obtained by restricting to Dubins-like paths.
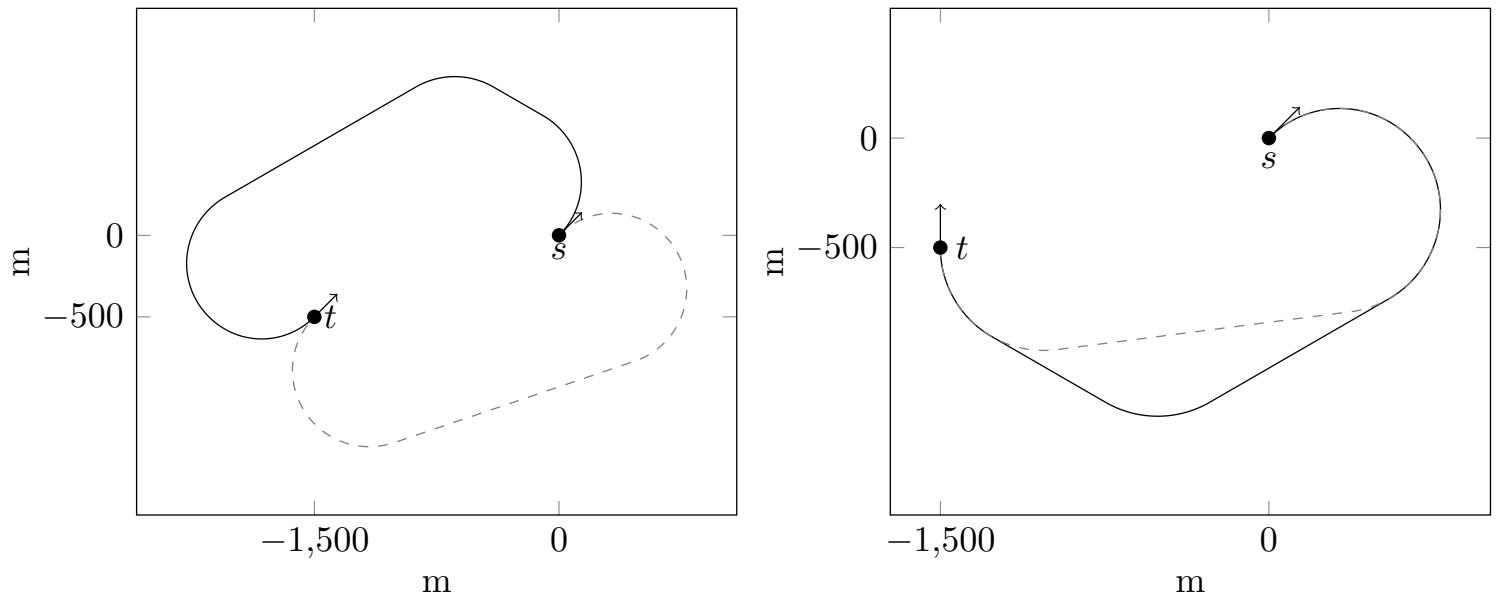

Figure 14: Examples of fastest-paths for the S175 containership in Sea State 7 compared to the Dubins-like fastest-paths (in dashed).

\subsection{Vehicle moving in constant and uniform field}

It was mentioned in the introduction that the algorithms developed in Dolinskaya and Maggiar 2012 and this paper represent a wider framework of which many extensions of the Dubin's problem are particular cases. One such extension is that of the Dubin's problem in a constant and uniform flowfield. This problem arises naturally in many application, notably in the case of Unmanned Aerial Vehicles (UAVs), which are very susceptible to the surrounding wind conditions. Thus, it is paramount to take the effects of the wind into account in order to properly plan the UAVs' paths. This problem was first tackled by McGee et al. 2005, who observed that it could be recast as a moving target problem in the absence of wind. They proved that the fastest paths in the presence of wind still had the same structure as the regular Dubin's paths, i.e., $\mathcal{C S C}$ or $\mathcal{C C C}$, although some properties satisfied by the solutions to the original problem had to be waived. The solution they propose was then to iteratively solve regular Dubin's problems until convergence. Techy and Woolsey 2009] offered a different approach by working directly in the ground referential instead of the inertial referential, exploiting the fact that the sharpest turns of a planar vehicle subject to a constant and uniform flowfield are described by trochoids Rysdyk, 2007. It is shown in this section how Algorithm 1 can be used to solve this problem. While this particular application is not a new contribution to the field, it 
serves as an illustrative example of how the setting in which this paper works is a generalization of previous problems and has a broader range of applications, which can potentially trigger the use of more realistic and complex vehicle models.

\subsubsection{Notation and speed and minimum-turning radius functions}

The notation and some results used are closely related to those presented by Techy and Woolsey 2009]. In the presence of a flowfield, it is important to distinguish between the relative and ground (or inertial) reference frames. The relative reference frame is mobile with respect to the ground reference frame and its movement is dictated by the flowfield. In the present case, a constant and uniform flowfield is considered. Without loss of generality, it can be chosen parallel to and directed as the $x$ axis of the absolute reference frame. Any other setting can be transformed into this one by rotating the axis. Let $W$ be the (signed) speed of the flow.

The trajectory of the vehicle can be equivalently described in either reference frames. In the relative reference frame the direction of the trajectory at time $t$ is denoted $\theta(t)$ and corresponds to the heading (steering) angle of the vehicle. Let $V_{r}(\theta)$ be its speed function, which is constant according to the Dubin's car model: $V_{r}(\theta)=V_{r}, \forall \theta \in S^{1}$. Similarly, let $\chi$ be the course angle of the vehicle in the ground reference frame and $V_{g}(\chi)$ its speed function.

Algorithm 1 operates in the ground reference frame, and it is necessary to derive the expressions of $V_{g}(\chi)$ and $R_{g}(\chi)$ in order to be able to apply it. It can be shown that these functions are expressed as:

$$
V_{g}(\chi)=W \cos \chi+\sqrt{V_{r}^{2}-W^{2} \sin ^{2} \chi}, \quad R_{g}(\chi)=R\left(\frac{V_{r}(\chi)^{3}}{V_{r}\left(V_{r}^{2}-W^{2}+W V_{g}(\chi) \cos \chi\right)}\right) .
$$

\subsubsection{Numerical examples}

Having derived the expressions for the speed and minimum-turning functions in $(19)$, Algorithm 1 may now be used to replicate the examples in McGee et al. 2005, which correspond to the following configurations: 1) $\theta_{s}=\pi / 2,\left(x_{t}, y_{t}, \theta_{t}\right)=(-1.5,2,0), V_{r}=1, R=1$ and $W=-0.5$, and 2) $\theta_{s}=\pi / 4,\left(x_{t}, y_{t}, \theta_{t}\right)=(5,1, \pi)$, $V_{r}=1, R=1$ and $W=0.5$. It should be noted that the speed polar plot corresponding to $V_{g}(\chi)$ is convex for it is essentially obtained by shifting the speed polar plot of of $V_{r}(\theta)$, which is a circle. Consequently, we do not need to apply the entirety of Algorithm 1, but only the step solving for Dubins-like paths, i.e. Algorithm 1 from Dolinskaya and Maggiar 2012.

The fastest paths obtained for these examples are presented in Figure 15, and it can be observed that these results match the ones obtained in that paper.
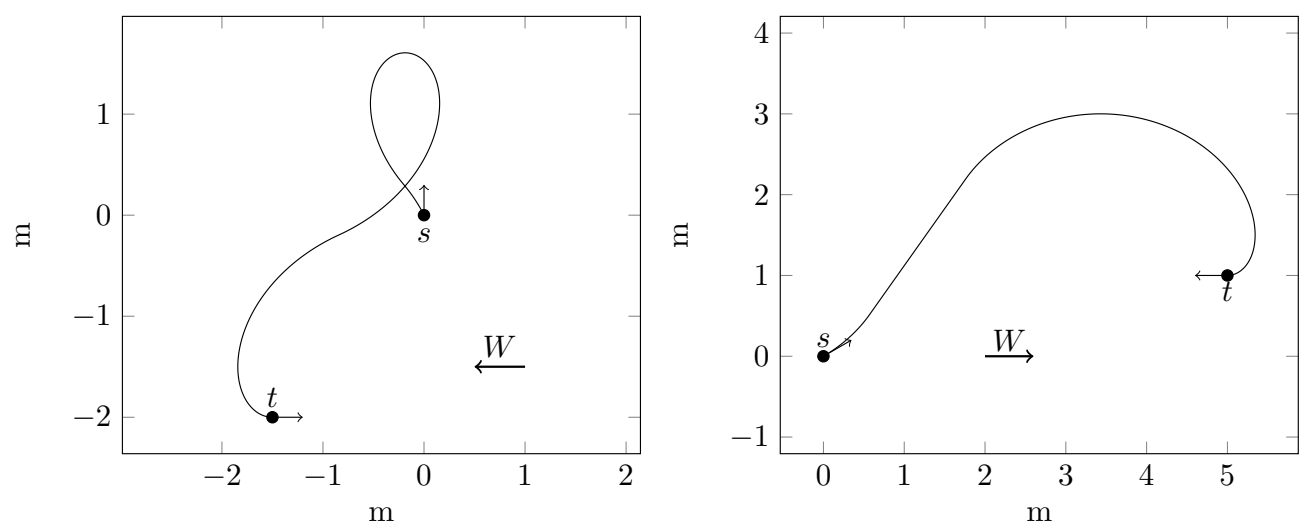

Figure 15: Fastest paths for the examples taken from McGee et al. 2005. 


\section{Conclusion}

This paper presented the implementation of an algorithm that computes the fastest path between an initial and a final configuration in an anisotropic medium. This represents a significant contribution since only the structure of optimal paths in anisotropic media had thus far been derived. In doing so, additional properties satisfied by optimal paths were obtained. In addition, it was shown that the direction-dependent framework generalizes some of the previous work, in particular, Dubins-like vehicles moving in constant and uniform wind.

The direction-dependent model studied in this paper represents a significant extension of the original Dubins car model, which assumes isotropic speed and minimum-turning radius. This development enables more physically realistic representations of several vehicles' motion, in particular in the field of unmanned vehicles. Some assumptions could further be relaxed in future work in order to generalize the model, for instance the assumption that the vehicle always travels at its maximum speed, which would keep improving the relevance and applicability of the results. Likewise, it might be interesting in some applications to free one or either of the extremal (initial or final) headings. Additionally, many properties of the optimal paths for the Dubins' vehicles have been explored in the literature, such as the synthesis of the fastest paths, and could also be investigated in the anisotropic case.

\section{Funding}

This work was supported in part by the Office of Naval Research through the Autonomous Vehicle Dynamic Navigation System grant (N00014-11-1-0516). 


\section{References}

L. E. Dubins. On curves of minimal length with a constraint on average curvature, and with prescribed initial and terminal positions and tangents. American Journal of Mathematics, 79(3):497-516, 1957.

I. S. Dolinskaya and A. Maggiar. Time-optimal trajectories with bounded curvature in anisotropic media. The International Journal of Robotics Research, 31(14):1761-1793, 2012. doi: 10.1177/0278364912458464.

J. A. Reeds and L. A. Shepp. Optimal paths for a car that goes both forwards and backwards. Pacific Journal of Mathematics, 145(2):367-393, 1990.

J. Sussmann and G. Tang. Shortest paths for the Reeds-Shepp car: a worked out example of the use of geometric techniques in nonlinear optimal control. Technical report, Dept. of Mathematics, Rutgers University, Piscataway, NJ, 1991.

J. D. Boissonnat, A. Cerezo, and J. Leblond. Shortest paths of bounded curvature in the plane. Journal of Intelligent and Robotic Systems, 11(1):5-20, 1994.

X. N. Bui, J. D. Boissonnat, P. Soueres, and J.-P. Laumond. Shortest path synthesis for Dubins nonholonomic robot. 1994 IEEE International Conference on Robotics and Automation, 1:2-7, 1994.

P. Soueres and J. D. Boissonnat. Optimal trajectories for nonholonomic mobile robots. In J.P. Laumond, editor, Robot motion planning and control, pages 93-170. Springer, 1998.

T. G. McGee, S. Spry, and J. K. Hedrick. Optimal path planning in a constant wind with a bounded turning rate. In AIAA Guidance, Navigation, and Control Conference and Exhibit, 2005.

R. L. McNeely, R. V. Iyer, and P. R. Chandler. Tour planning for an unmanned air vehicle under wind conditions. Journal of Guidance, Control, and Dynamics, 30(5):1299-1306, 2007.

S. Hima and Y. Bestaoui. Time-optimal paths for lateral navigation of an autonomous underactuated airship. In AIAA Conference on Navigation, Guidance and Control, Austin, Texas, 2003.

I. S. Dolinskaya, M. Kotinis, M. G. Parsons, and R. L. Smith. Optimal Short-Range Routing of Vessels in a Seaway. Journal of Ship Research, 53(3):121-129, 2009.

A. B. Philpott and A. Mason. Optimising yacht routes under uncertainty. In Proceedings of the 15th Chesapeake Sailing Yacht Symposium, Annapolis, MD, 2001.

A. J. Chang, M. Brazil, J. H. Rubinstein, and D. A. Thomas. Curvature-constrained directional-cost paths in the plane. Journal of Global Optimization, 53(4):663-681, May 2012. doi: 10.1007/s10898-011-9730-1.

A. J. Chang, M. Brazil, J. H. Rubinstein, and D. A. Thomas. Optimal curvature-constrained paths for general directional-cost functions. 2011. doi: 10.1007/s11081-011-9180-0.

H. Wang, Y. Chen, and P. Soueres. A Geometric Algorithm to Compute Time-Optimal Trajectories for a Bidirectional Steered Robot. IEEE Transactions on Robotics, 25(2):399-413, 2009.

L. Techy and C. A. Woolsey. Minimum-Time Path Planning for Unmanned Aerial Vehicles in Steady Uniform Winds. Journal of Guidance, Control, and Dynamics, 32(6):1736-1746, November 2009. doi: $10.2514 / 1.44580$.

A. J. Chang. Optimal Curvature and Gradient-constrained Paths with Anisotropic Costs. PhD thesis, University of Melbourne, 2011.

J. Nocedal and S. J. Wright. Numerical optimization. Springer, second edition, 2006.

B. K. Youse. Calculus with Analytical Geometry. Holt, Rinehart and Winston, 1978.

F. Fabricius-Bjerre. On the double tangents of plane closed curves. Mathematica Scandinavia, 11:113-116, 1962. 
W. Rudin. The Principles of Mathematical Analysis. International Series in Pure \& Applied Mathematics. McGraw-Hill, third edition, 1976.

R. Rysdyk. Course and Heading Changes in Significant Wind. Journal of Guidance, Control, and Dynamics, 30(4):1168 - 1171, 2007. 